\title{
Performance Results for Sensor Assignment Problem as Solved on a Multi-Node Cluster
}

Process-Constrained Data Analytics for Sensor Assignment and Calibration

Nuclear Science and Engineering Division 


\section{About Argonne National Laboratory}

Argonne is a U.S. Department of Energy laboratory managed by UChicago Argonne, LLC under contract DE-AC02-06CH11357. The Laboratory's main facility is outside Chicago, at 9700 South Cass Avenue, Argonne, Illinois 60439. For information about Argonne and its pioneering science and technology programs, see www.anl.gov.

\section{DOCUMENT AVAILABILITY}

Online Access: U.S. Department of Energy (DOE) reports produced after 1991 and a growing number of pre-1991 documents are available free at OSTI.GOV (http://www.osti.gov/), a service of the US Dept. of Energy's Office of Scientific and

Technical Information.

Reports not in digital format may be purchased by the public from the National Technical Information Service (NTIS):

U.S. Department of Commerce

National Technical Information

Service 5301 Shawnee Rd

Alexandria, VA 22312

www.ntis.gov

Phone: (800) 553-NTIS (6847) or (703) 605-6000

Fax: (703) 605-6900

Email: orders@ntis.gov

Reports not in digital format are available to DOE and DOE contractors from the Office of Scientific and Technical Information (OSTI):

U.S. Department of Energy

Office of Scientific and Technical Information

P.O. Box 62

Oak Ridge, TN 37831-0062

www.osti.gov

Phone: (865) 576-8401

Fax: (865) 576-5728

Email: reports@osti.gov

\section{Disclaimer}

This report was prepared as an account of work sponsored by an agency of the United States Government. Neither the United States Government nor any agency thereof, nor UChicago Argonne, LLC, nor any of their employees or officers, makes any warranty, express or implied, or assumes any legal liability or responsibility for the accuracy, completeness, or usefulness of any information, apparatus, product, or process disclosed, or represents that its use would not infringe privately owned rights. Reference herein to any specific commercial product, process, or service by trade name, trademark, manufacturer, or otherwise, does not necessarily constitute or imply its endorsement, recommendation, or favoring by the United States Government or any agency thereof. The views and opinions of document authors expressed herein do not necessarily state or reflect those of the United States Government or any agency thereof, Argonne National Laboratory, or UChicago Argonne, LLC. 
ANL/NSE-21/50

\section{Performance Results for Sensor Assignment Problem as Solved on a Multi-Node Cluster}

Process-Constrained Data Analytics for Sensor Assignment and Calibration

prepared by

Tim Nguyen, Hubert Ley and Richard B. Vilim

Nuclear Science and Engineering Division, Argonne National Laboratory

Yuxuan Liu and Brendan Kochunas

Nuclear Engineering \& Radiological Sciences, University of Michigan

August 31, 2021 


\section{ABSTRACT}

An earlier report described a procedure for optimal sensor set selection and its implementation on a computational cluster. This new and innovative capability was developed to facilitate a reduction in operations staffing levels to improve plant economics. By automating surveillance and maintenance tasks through early detection of degrading sensors and equipment, staff can be more efficiently deployed. The method uses automated reasoning and domain knowledge in the form of the conservation equations to infer from plant measurements the state of equipment health. Inclusion of domain knowledge addresses the problem that exists with pure data-driven methods that there are no rigorous guidelines for determining what constitutes an adequate sensor set. Formalizing the procedure for sensor set selection as we have done results in a more reliable and explainable diagnosis of plant equipment health. Importantly, from the standpoint of the plant owner, personnel are provided with an early and explicit diagnosis of an equipment problem. That in principle automates the process and eliminates having to send personnel into the plant to find the cause as typically occurs when a data-driven method detects an anomaly.

In this report we describe first results obtained using a computational cluster to solve the sensor set selection problem as framed above. The case described addresses the problem of equipment health monitoring in the high-pressure (HP) feedwater system of a pressurized light water reactor as seen through the eyes of our collaborating utility partner. Maintenance of this system can amount to millions of dollars per year if equipment health issues go undiagnosed and lead to loss of function. On examining the potential that is inherent in the installed sensor set for diagnosing equipment health degradation, it was found that greater fault resolution capability can be achieved using a sensor set that is 20 percent fewer in number. The takeaway is that compared to the installed sensor set there exists a more strategic assignment of sensors that will furnish better health monitoring capability and with fewer sensors. Where the problem defies solution by manual inspection, as is the case here, one can be found by an algorithm. The solution was obtained in four hours using 30 computational cores.

The HP feedwater problem as posed above illustrates the added value of approaching the sensor selection problem as one amenable to algorithmic solution. This problem is of interest to advanced reactor designers and to utilities that are setting up remote monitoring and diagnostic centers. 


\section{TABLE OF CONTENTS}

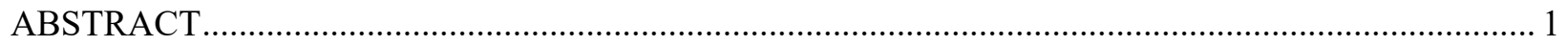

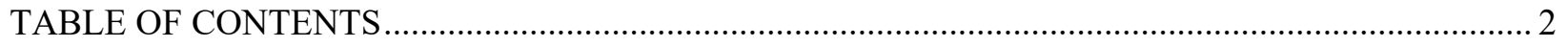

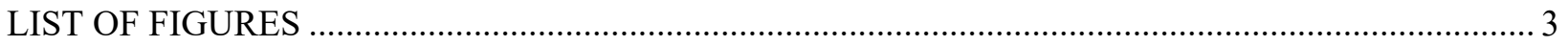

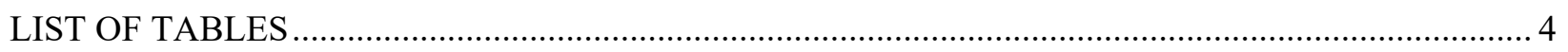

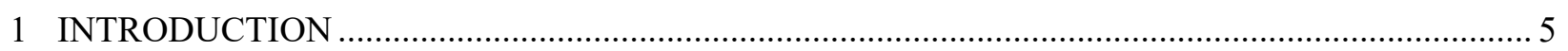

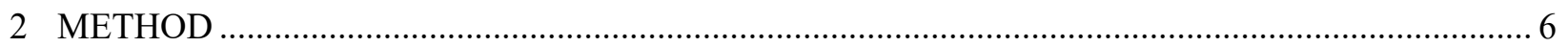

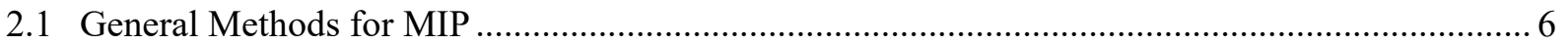

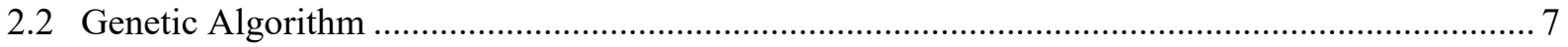

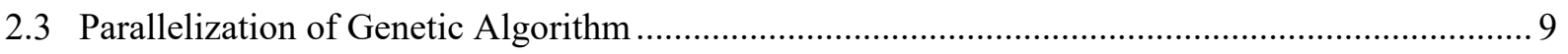

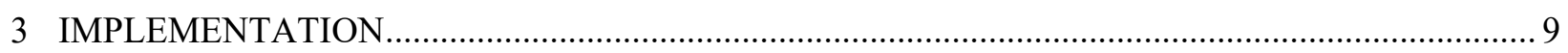

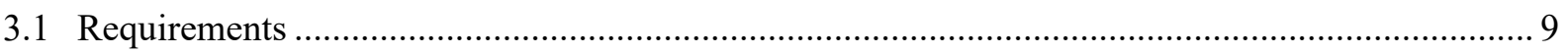

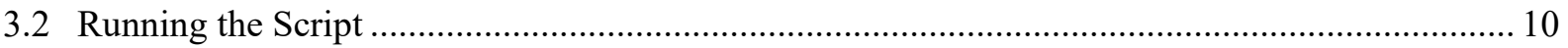

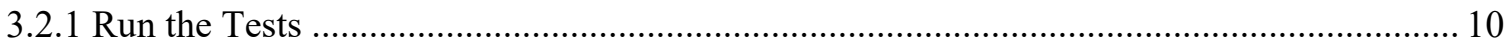

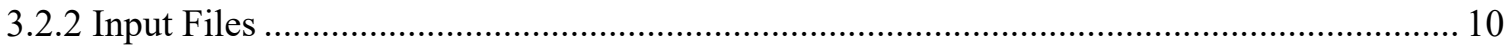

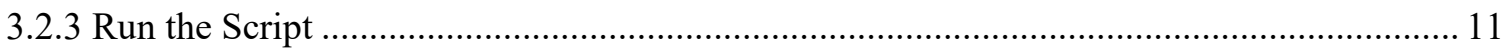

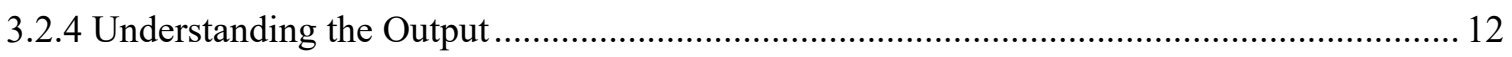

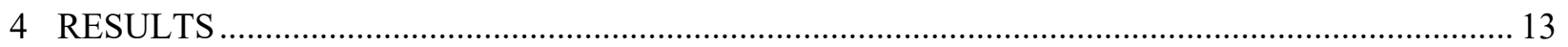

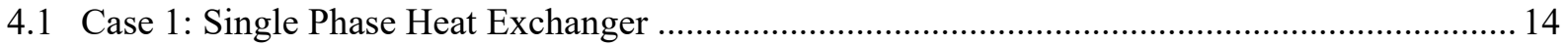

4.2 Case 2: Two Feedwater Heaters in Parallel .............................................................................. 15

4.3 Case 3: High Pressure Feedwater System ............................................................................... 19

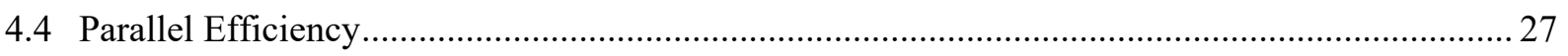

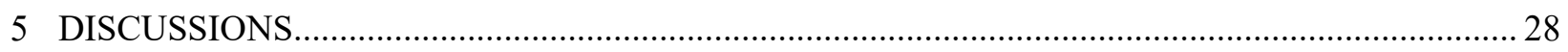

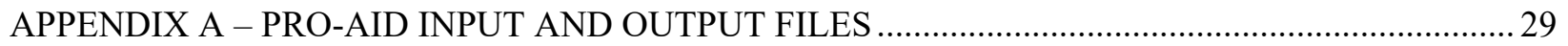

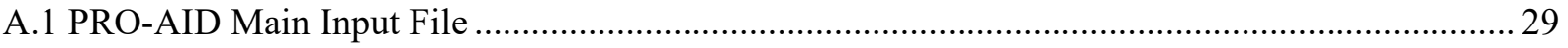

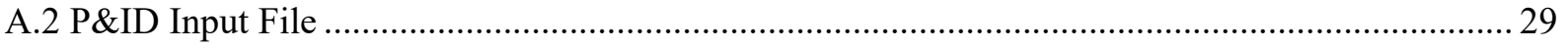

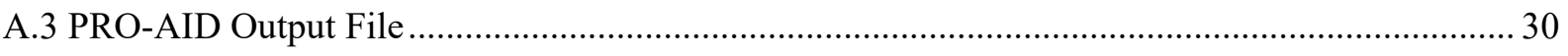

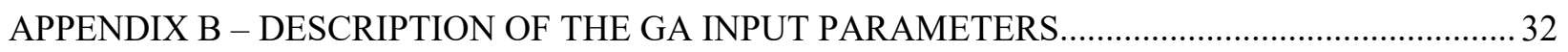

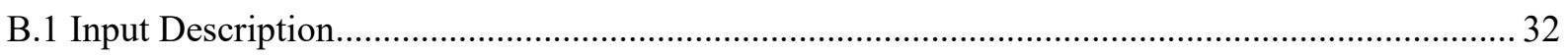

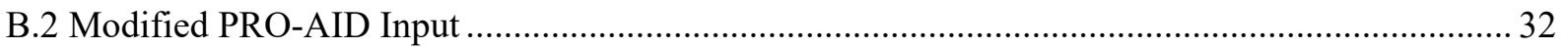

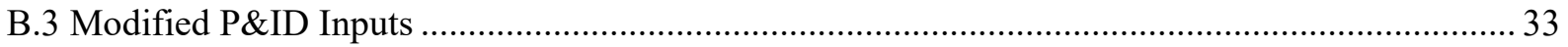

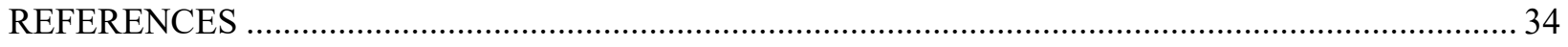




\section{LIST OF FIGURES}

Figure 1 Tree structure of branch-and-bound method …....................................................................... 7

Figure 2 Sequence of the GA for sensor assignment optimization ...................................................... 8

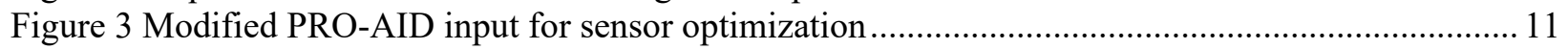

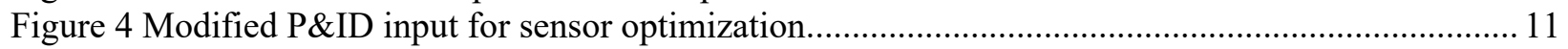

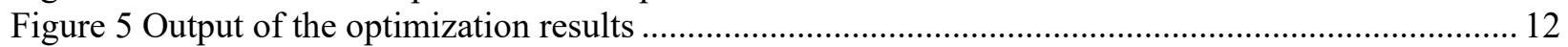

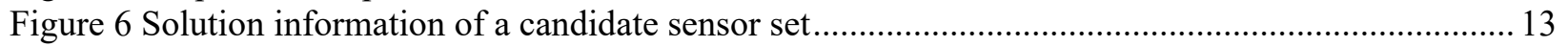

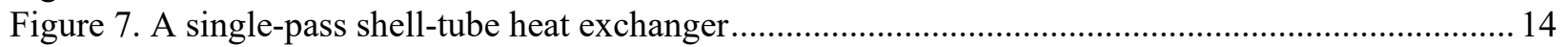

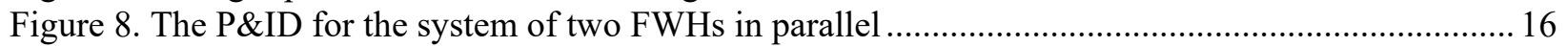

Figure 9. Sensor set requirement for a standalone FWH diagnostic model .............................................. 16

Figure 10. The optimal sensor set (green) and virtual sensors (red) created in PRO-AID for test case \#2 18

Figure 11. The P\&ID with the installed sensor set for the feedwater system in test case \#3.................... 21

Figure 12. Simplified P\&ID used in PRO-AID for test case \#3 ....................................................... 22

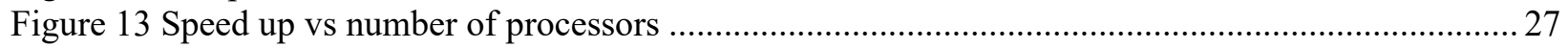




\section{LIST OF TABLES}

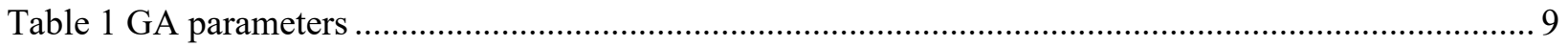

Table 2. Sensor cost and fault diagnosis scaling factor of the second case .......................................... 13

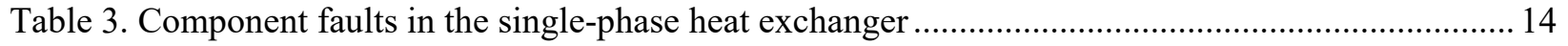

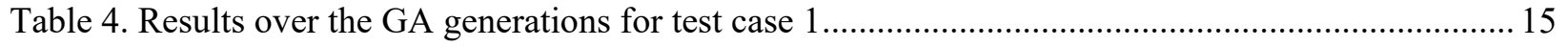

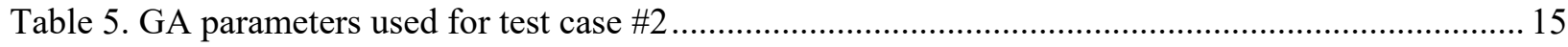

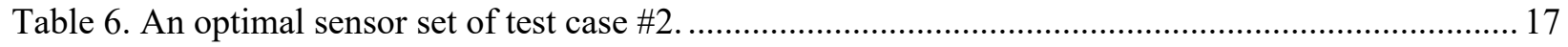

Table 7. Output summary of the selected optimal sensor set for test case \#2 ....................................... 17

Table 8. Available diagnostic models from the installed sensor set test case \#3 ..................................... 19

Table 9. Detectable component faults using the installed sensor set for test case \#3 ..............................20

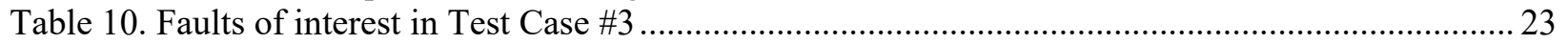

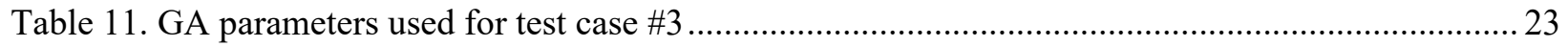

Table 12. Summary of the optimal sensor set for test case \#3 …........................................................24

Table 13. PRO-AID diagnostic models given the optimal sensor set for Test Case \#3 …........................25

Table 14. Comparisons of diagnostic capabilities of the installed sensor set and the optimal sensor set... 26

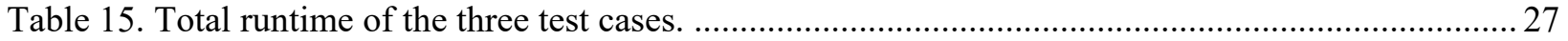




\section{INTRODUCTION}

The monitoring capabilities of commercial nuclear power plants that entered service in the early 1980's were designed with sensor sets that provided the most basic required functionality. There was a need to monitor plant conditions for maintaining reliable power production and for alarming when conditions move outside the range of normal operation. Compared to the monitoring capability being envisioned today for advanced reactor concepts, the sensor set in those plants is relatively limited. Factors such as the relatively high cost of communication by copper wire, and associated cable trays, compared to newer communication technologies available today limited the number of sensing points.

The picture has changed significantly since that time. New and more capable monitoring technologies are supported by advances in communication, sensing, computer hardware, and data processing algorithms. Fiber optic cable and wireless transmission with their low power and high bandwidth capability provide cost benefits that allow for larger sensor sets. New sensing modalities such as optical, acoustic, microwave, and chemical provide a richer set of process variables that can be monitored. Advances in software development tools enable the cost-effective implementation of sophisticated algorithms to make sense of data from a system and plant-wide perspective compared to that of a single-point alarm.

This project is addressing the problem of how to select a sensor set for equipment and sensor health monitoring for advanced O\&M tasks. Under this award, collaborating utilities have provided insight on how the advanced monitoring methods being developed can enable staffing and equipment cost reductions at nuclear power plants. Some of these findings include the following:

- Safety system sensors require periodic calibration. Being able to unobtrusively determine the calibration status could obviate the need for performing unnecessary instrument calibration.

- Safety system equipment requires periodic testing. Being able to unobtrusively determine performance status of equipment could provide additional safety credit.

- Lifetime extension applications are to include plans for the digital upgrade of balance-of-plant systems. Understanding how the selection of an upgraded sensor set contributes to improved monitoring and control can provide for more informed staffing reductions and reliable operation.

These findings are indicative of general industry needs and have helped to shape our requests for data from our utility collaborators.

An earlier report described a procedure for optimal sensor set selection and its implementation on a computational cluster. The method uses automated reasoning and domain knowledge in the form of the conservation equations to infer from plant measurements the state of equipment health. Inclusion of domain knowledge addresses the problem that exists with pure data-driven methods that there are no rigorous guidelines for determining what constitutes an adequate sensor set [1]. Formalizing the procedure for sensor set selection as we have done results in a more reliable and explainable diagnosis of plant equipment health. Importantly, from the standpoint of the plant owner, personnel are provided with an early and explicit diagnosis of an equipment problem. That in principle automates the process and eliminates having to send personnel into the plant the find the cause as typically occurs when a data-driven method detects an anomaly [2].

In this report we describe first results obtained using a computational cluster to solve the sensor set selection problem as framed above. The case described addresses the problem of equipment health monitoring in the high-pressure (HP) feedwater system of a pressurized light water reactor as seen through the eyes of our collaborating utility partner. Maintenance of this system can amount to millions of dollars per year if equipment health issues go undiagnosed and lead to loss of function. On examining the potential that is inherent in the installed sensor set for diagnosing equipment health degradation, it was found that greater fault resolution capability can be achieved using a sensor set that is 20 percent fewer in number. The take-away is that compared to the installed sensor set there exists a more strategic assignment of sensors 
that will furnish better health monitoring capability and with fewer sensors. Where the problem defies solution by manual inspection, as is the case here, one can be found by an algorithm. The solution was obtained in four hours using 30 computational cores.

The HP feedwater problem as posed above illustrates the added value of approaching the sensor selection problem as one amenable to algorithmic solution. This problem is of interest to advanced reactor designers and to utilities that are setting up remote monitoring and diagnostic centers.

This report is organized as follows. Section 2 summarizes the feature and capabilities of the algorithms that were developed in the first two years of the project. Section 3 describes the implementation of the optimization algorithms and the integration with the fault diagnostic code PRO-AID. Results of three test cases and an analysis of the computational performance are provided in Section 4. Finally, Section 5 discusses the performance results.

\section{METHOD}

Optimizing the assignment of sensors may be classified as a mixed-integer programming (MIP) problem where the decision variables - whether to set a sensor in a specific location - is 0 or 1 . The optimization objective is to minimize the cost function that represents the cost of satisfying a set of diagnostic objectives for a given set of sensors. The cost function in principle should reflect the degree of fault spatial localization achieved, the quality of diagnosis measured in terms of the sensitivity to fault severity, and the cost of procuring and installing the sensor set. The cost varies with the number and types of sensors in the sensor set. The cost function is minimized to obtain the highest value sensor set - the sensor set that best delivers on the required fault diagnosis capabilities while considering cost. In this work, we evaluate a simplified cost function that includes the cost of the sensor and accounts for whether a required diagnosis is in fact made, as defined in the following equation [3],

$$
\min f(\vec{k})=\left(\overrightarrow{C_{I}}+\overrightarrow{C_{P}}\right) \cdot \vec{k}-\sum_{l} \alpha_{l} D_{l}(\vec{k})
$$

where

$$
\begin{aligned}
& \begin{array}{ll}
\vec{k} & =\text { vector of binary variables for whether to include each of the sensors } \\
\overrightarrow{C_{I}} & =\text { vector of cost for installing sensors } \\
\overrightarrow{C_{P}} & =\text { vector of cost for procuring sensors } \\
D_{l}(\vec{k}) \quad=\text { diagnosis status }(0 \text { or } 1) \text { for fault } l \text { using the sensor set } \vec{k} \\
\alpha_{l} \quad=\text { scaling factor }
\end{array}
\end{aligned}
$$

The set of sensors that minimizes the cost function is optimal. If a successful diagnosis of a specific fault needs to be guaranteed, the scaling factor of the fault can be set to a large value to inform the solver to select the sensor set that can diagnose the fault. Rather than an explicit function, the diagnosis status $D_{l}(\vec{k})$ is determined by running the diagnostic code PRO-AID [4, 5].

\subsection{General Methods for MIP}

A general optimization problem can be classified in different ways [6, 7], e.g., convex or non-convex, linear or non-linear, continuous or discrete variables, etc. There are a variety of deterministic optimization 
solvers that target different kinds of problems, as introduced in Ref. [8]. The MIP problems are generally solved using a branch-and-bound algorithm [7]. The first step is to remove the integer restrictions of the variables. The resulting problem is called a "relaxation" of the original MIP. Solving this problem with general linear or non-linear programming methods yields the optimized values with no guarantee of integers. Then sub-problems are created by adding integer bounds around the optimized value as constraints to "branch out" the problem. For each sub-problem, the same process can be applied until integer optimized values are achieved; this normally forms a branch-and-bound search tree as shown in Figure 1. Nevertheless, the relaxations need to be solved by deterministic programming solvers. These solvers normally require the knowledge of derivatives of the objective function. In our problem the derivatives are not available due to the $D_{l}(\vec{k})$ term; unless a surrogate model is developed. With the intention of directly calling PRO-AID for fault diagnoses, derivative-free approaches are desired for this work.

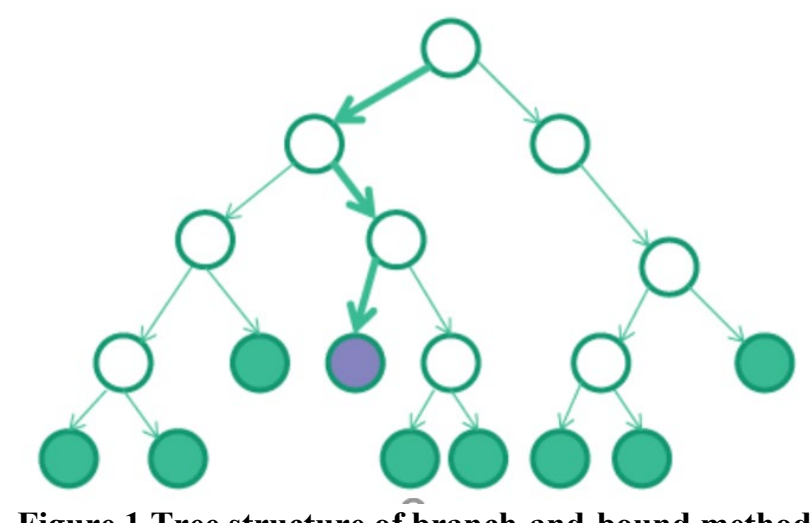

Figure 1 Tree structure of branch-and-bound method

\subsection{Genetic Algorithm}

The genetic algorithm (GA) is one of the evolutionary algorithms that mimic the natural selection process taking place in natural evolution [9]. Unlike the deterministic methods, the GA does not rely on derivative information to guide them toward a local maximum/minimum. Instead, the search is guided solely by the fitness value, i.e., the cost function. The method employs the selection, crossover and mutation operators on a set of 'chromosomes' that characterize the individuals. Intuitively the GA proceeds by creating successive generations of better and better individuals by applying these simple operations. The fitness value is used to rank individuals depending on their relative suitability for the problem being solved.

For the sensor optimization problem, the chromosome of an individual is an array of binary variables of whether to set a sensor in each of the predefined locations. The search starts by generating an initial population of individuals (chromosomes). This first population must offer a wide diversity of genetic materials. The gene pool should be sufficiently large to statistically cover the search space. The initial population is generated randomly. Then the GA loops over an iteration process to make the population evolve. Each iteration consists of the following steps:

- Selection: The first step consists in selecting individuals for reproduction. This selection is done randomly with a probability depending on the relative fitness of the individuals so that best ones are often chosen for reproduction than poor ones.

- Reproduction: In the second step, offspring are bred by the selected individuals. For generating new chromosomes, the algorithm can use both crossover and mutation.

- Evaluation: Then the fitness of the new chromosomes is evaluated. 
- Replacement: During the last step, individuals from the old population are killed and replaced by the new ones.

Figure 2 shows the flowchart of the GA iteration for the sensor assignment problem. The calculation is initialized by modified PRO-AID inputs that define the search space of the sensors. When evaluating an individual, the $D_{l}(\vec{k})$ in Eq. (1) requires calling PRO-AID to obtain the diagnoses results. This is currently realized by file coupling. The inputs for the sensor set being evaluated are generated on the fly, and the results of PRO-AID are processed to obtain $D_{l}(\vec{k})$.

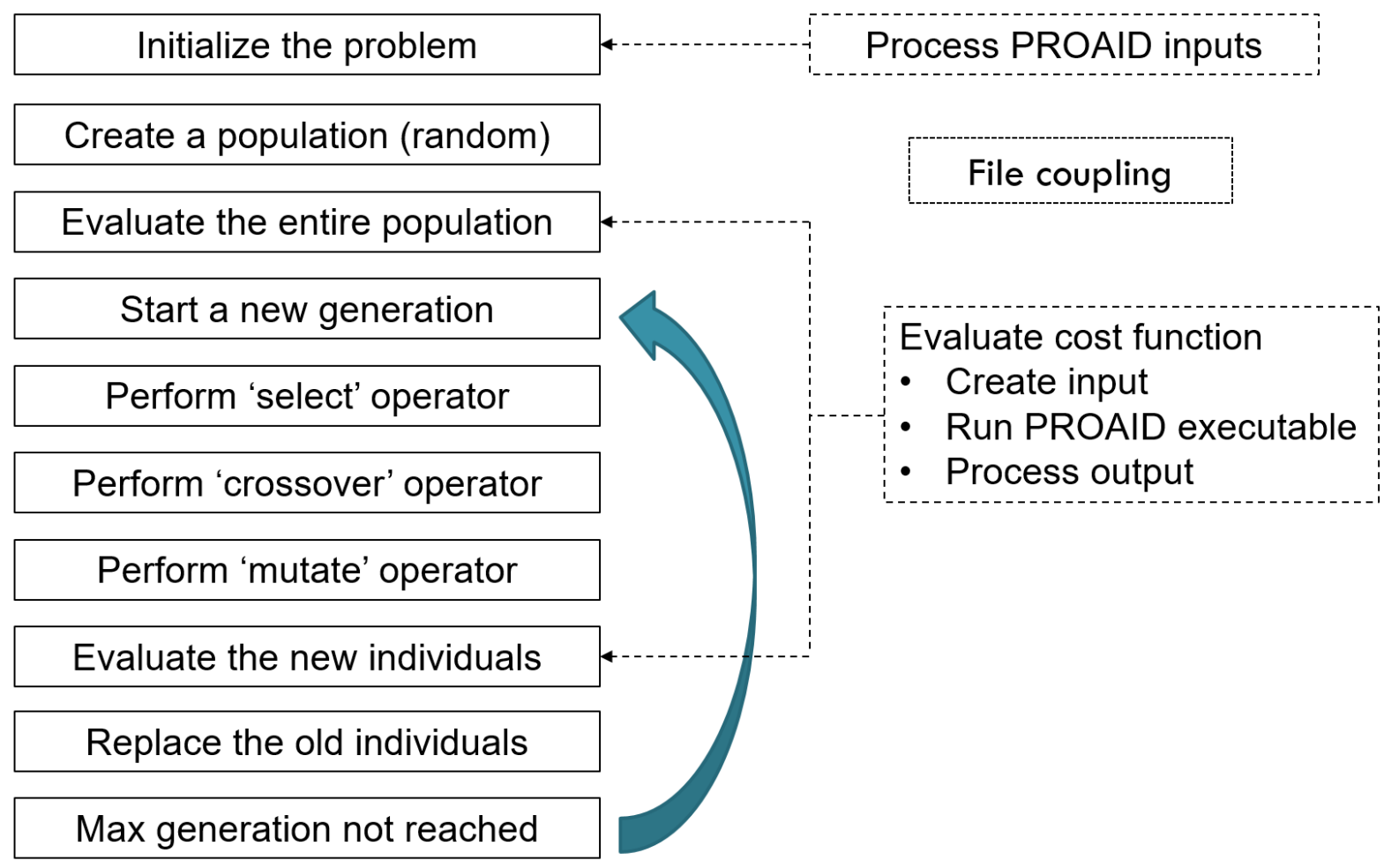

Figure 2 Sequence of the GA for sensor assignment optimization

Table 1 provides the GA parameters involved in the optimization process. These parameters can be tuned to improve the convergence of a specific problem. For the initial implementation, these parameters are provided as user inputs. 
Table 1 GA parameters

\begin{tabular}{|l|c|c|}
\hline \multicolumn{1}{|c|}{ Parameter } & $\begin{array}{c}\text { Typical value for general GA } \\
\text { applications }\end{array}$ & $\begin{array}{c}\text { Recommended for sensor } \\
\text { optimization problem }\end{array}$ \\
\hline Number of individuals & Depend on the search space & $100 \sim 2000$ \\
\hline Number of generations & Depend on the search space & $10 \sim 20$ \\
\hline Tournament size for selection & $3-5$ & $3-5$ \\
\hline Crossover percentage on population & $0.5-1$ & $0.5-1$ \\
\hline Mutation percentage on population & $<0.5$ & $0.1-0.2$ \\
\hline Mutation probability to flip a gene & $<0.1$ & $0.01-0.1$ \\
\hline
\end{tabular}

\subsection{Parallelization of Genetic Algorithm}

There are several variations of the GA parallelization approach. The main idea is to partition either the whole population or the evaluation of individuals' fitness [9]. In the population partitioning, the population is divided into a number of subpopulations, which are separated from one another, and individuals compete only within a subpopulation. An additional operator called migration is used to move the individuals from one subpopulation to another. In the evaluation partitioning, a single population is used, and the evaluations of individuals are done in parallel.

With a runtime profiling of the first test problem that will be discussed in Section 4, it is identified that running PRO-AID to obtain diagnoses for evaluating the individuals' fitness dominates the total runtime ( $>99 \%$ of total runtime) of our optimization calculation. Therefore, the evaluation partitioning is used in this work with the manager-worker parallelization scheme. A worker requires only the knowledge of the individuals being evaluated, so no sophisticated communication is needed except for passing the individual chromosomes from the manager to the workers and then the fitness values from the workers to the manager. The operators that may alter the individuals such as selection, crossover and mutation are still done by the manager process. The number of individuals assigned to a processor for evaluation may be static or dynamic. An evenly distributed number of individuals are used in this work because the variation of PRO-AID runtimes becomes small unless the number of individuals per processor is very small (in this case, the runtime is no longer a concern).

\section{IMPLEMENTATION}

A Python script was developed to drive the optimization. It reads the modified PRO-AID and P\&ID inputs to obtain the sensor search space as well as the cost data. The Distributed Evolutionary Algorithms in Python (DEAP) package is used to perform the GA. Parallelization is realized behind the scenes through the built-in interfaces of DEAP to distribute the work of individual evaluations. The standard PRO-AID and P\&ID inputs are created when evaluating an individual. When the GA loops are completed, the statistics of each generation and the best candidates of the final generation are edited. This section describes the use of the script.

\subsection{Requirements}

Several software packages are used in the script:

- $D E A P$ (required) is a novel evolutionary computation framework that seeks to make algorithms explicit and data structures transparent, as opposed to the more common black box type of frameworks [10]. The aim is to provide a toolbox that encourages users to write their own evolutionary algorithms, explicitly controlling every aspect of the evolutionary process: data types, fitness measures, population initialization, operators, evolutionary loop, etc. It also 
incorporates easy parallelism where the implementation details like synchronization and load balancing are behind the scene. To install $D E A P$ using pip,

$$
\text { python -m pip install deap }
$$

- multiprocessing is required for python (version $<2.7$ ) to run the optimization in parallel. multiprocessing [11] is a package that supports spawning processes using an API similar to threads. multiprocessing has been distributed in the standard library since python 2.7 and above.

- $\quad P R O-A I D$ executable is required to evaluate the cost function when running the optimization. An environment variable 'PROAID_EXE_PATH' needs to be set for the script to find the executable, e.g.,

$$
\text { export PROAID_EXE_PATH=<path_to_proaid }>
$$

Alternatively, the script can be coupled directly with the PRO-AID source.

- pytest is optional for testing the optimization script. Alternatively, users can run the testing script directly. Details will be given in the next section.

\subsection{Running the Script}

\subsubsection{Run the Tests}

Two scripts are provided in the InverseSnesor repository, GAInverseSensor.py and test_GAInverseSensor.py. Along with the tests are the inputs in the folder/tests. The first step is to run the tests to ensure all the required packages are properly installed. Two ways are provided to run the test,

- Using pytest framework [12]. pytest is a Python-based testing framework, which is used to write and execute test codes. If pytest package is installed, user can simply do the following command in the root directory of InverseSensor,

$$
\text { pytest }
$$

- Alternatively, user can run the test script directly if pytest package is not available,

$$
\text { python test_GAInverseSensor.py }
$$

\subsubsection{Input Files}

The inputs of the program include modified PRO-AID and P\&ID inputs with the costs of sensors, scaling factors of fault diagnoses and optimization options. Details on the PRO-AID input and output files are provided in Appendix A. An example of the modified main PRO-AID input is shown in Figure 3. The two blue blocks are the data added to the original PRO-AID input. The first block includes the 'gain' (scaling factor $\alpha_{l}$ ) of successfully diagnosing a fault. The second block of data are the user options for the GA optimization. A detailed description of the inputs is included in Appendix B. Figure 4 shows an example of the modified P\&ID input. Only Block 3 of the input is included because this is the only place to be changed. The description of these data is also included in Appendix B. 


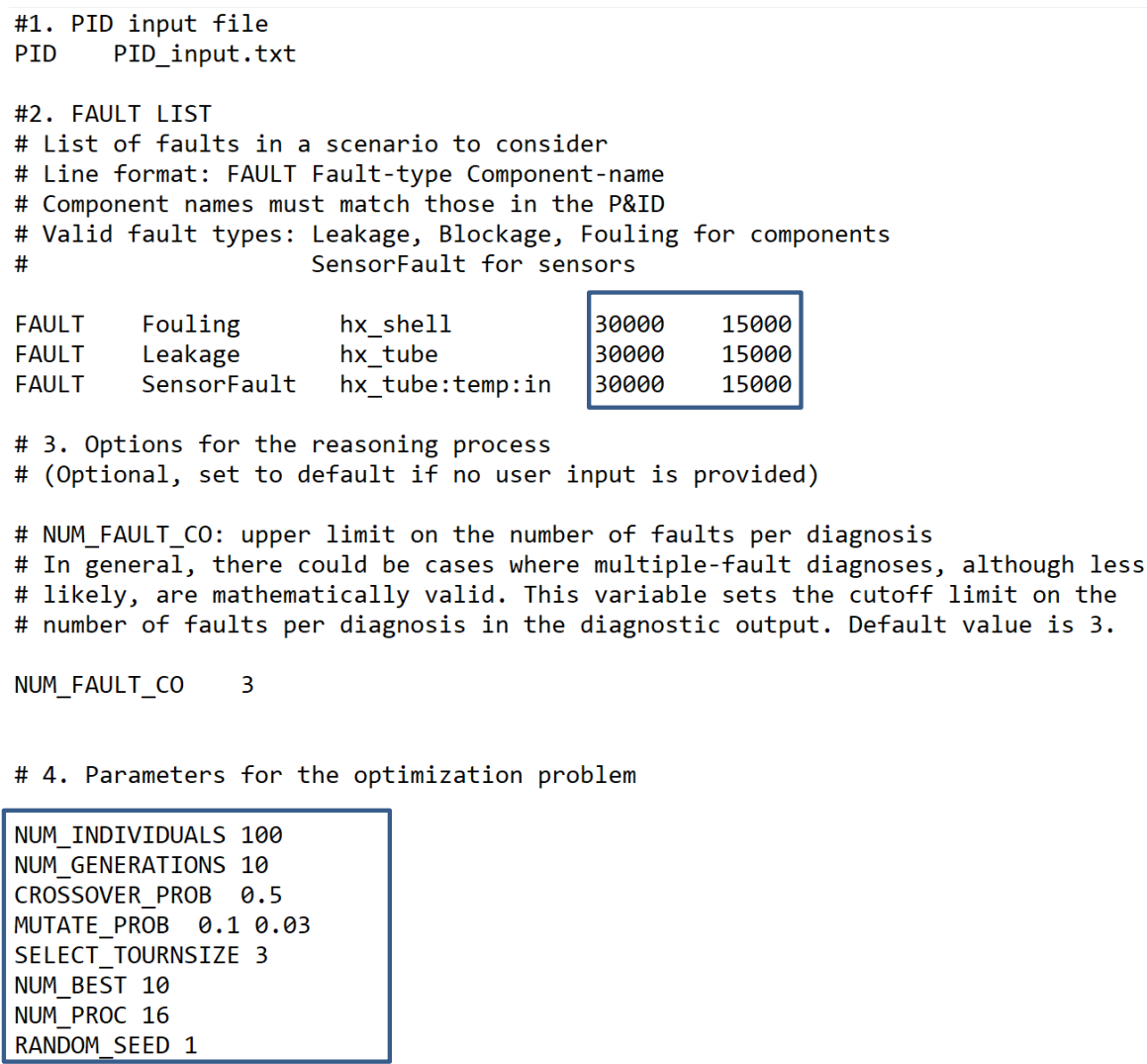

Figure 3 Modified PRO-AID input for sensor optimization

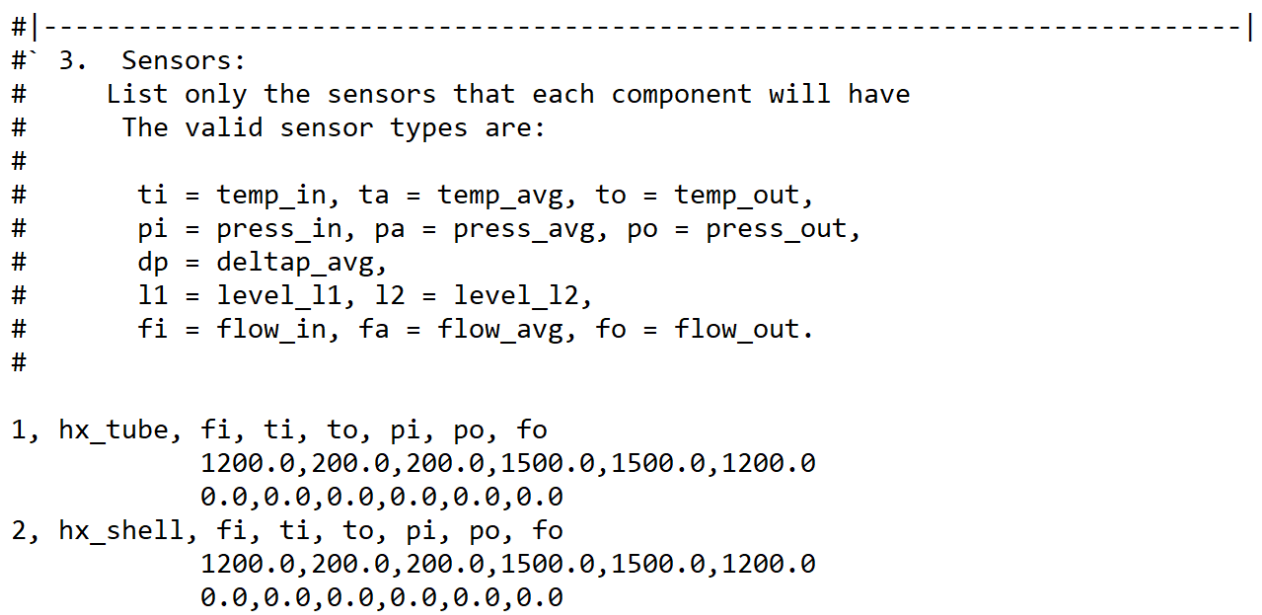

Figure 4 Modified P\&ID input for sensor optimization

\subsubsection{Run the Script}

To run the script, the location of the modified PRO-AID input is taken as the only argument. The following command will run the optimization script,

python GAInverseSensor.py <input.txt $>$ 
As usual, the location of the modified P\&ID input is given in the PRO-AID input. To run the GA in parallel with multiprocessing, the input option NUM_PROC need to be specified (larger than 1).

\subsubsection{Understanding the Output}

When running the script, the terminal printouts are mostly from executing PRO-AID. Once the calculation is done, the summary results can be found in results_x.out. ' $\mathrm{x}$ ' is the random number appended in the summary file name to avoid overriding the results since we normally run the same problem with a few random numbers. Figure 5 shows a sample summary file of 10 individuals run for 2 generations. Statistics are shown for each generation. The number of evaluated individuals show the total number of evaluation function called during the optimization. The GA parameters used in the calculation are also included, followed by the best candidates and best unique candidates from the last generation. The array of binaries encodes the sensor assignment. The corresponding PRO-AID and P\&ID inputs and PRO-AID output are all kept for further information. For example, the best \#1 will have files PID_011001111000.txt, input fn_011001111000.txt and output fn_011001111000.txt. Note the ' $\mathrm{n}$ ' in the last two file names is index number $(0,1,2, \ldots)$ of the faults. Each fault is run independently with PRO-AID to obtain the diagnoses. Also the sensor selection and fault diagnoses are edited into a solution file for every unique candidate in the best list. For example, sol_rand_0_rank1_011001111000.txt is generated for this case to show the solution of the best unique candidate (rank $1^{\text {st }}$ ) with random number 0 , as shown in Figure 6.

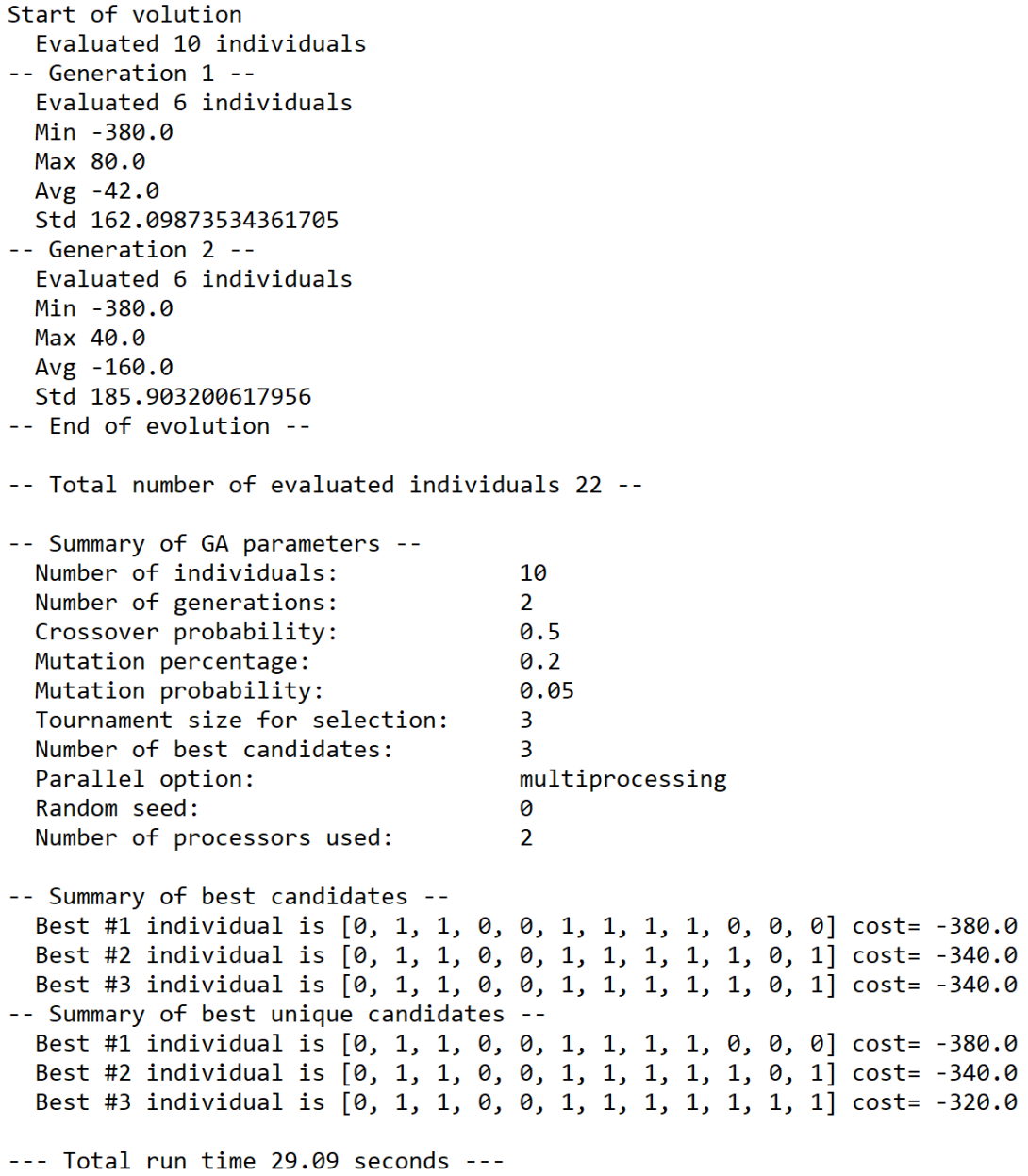

Figure 5 Output of the optimization results 


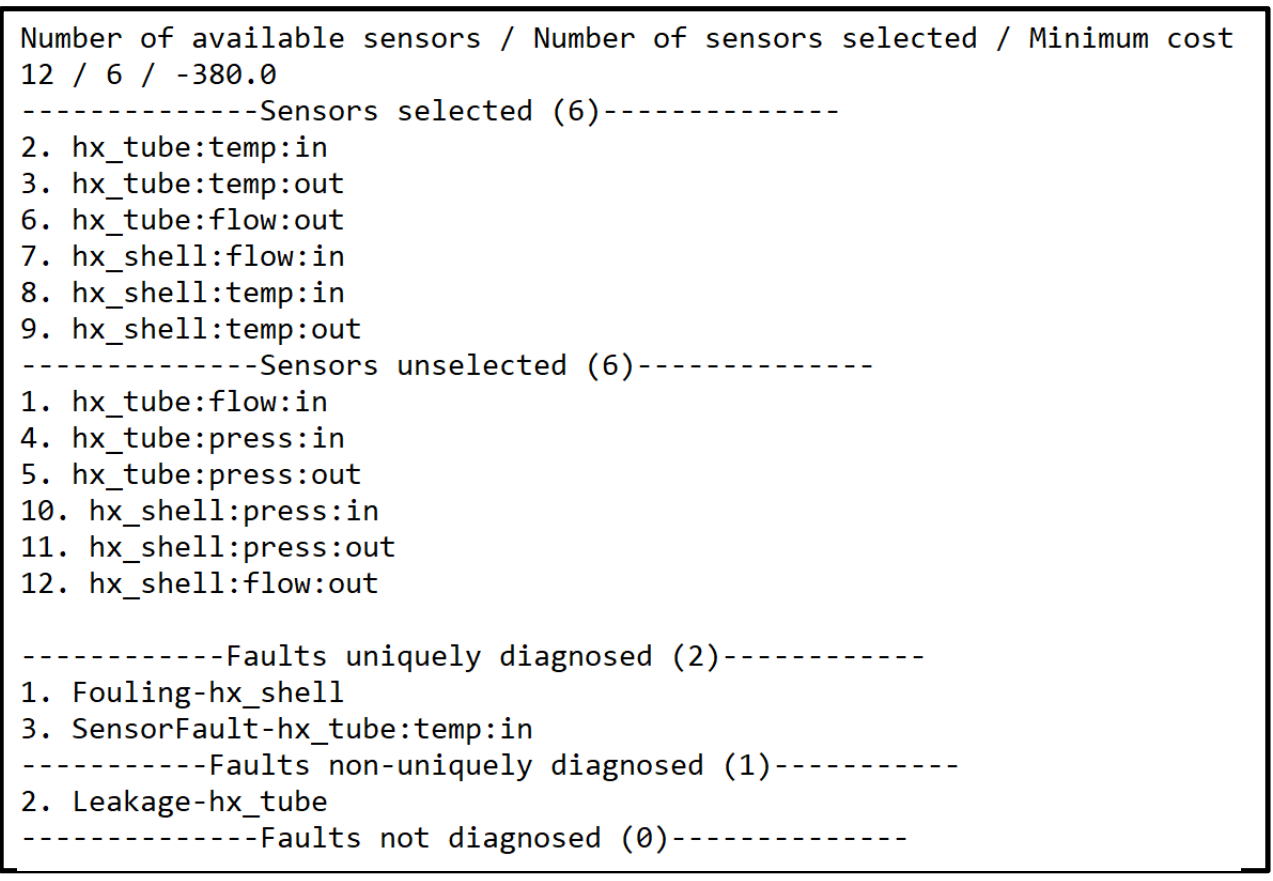

Figure 6 Solution information of a candidate sensor set

\section{RESULTS}

We considered three test cases in the increasing order of complexity to test the GA implementation:

- Test case 1: A standalone single-phase heat exchanger.

- Test case 2: Two feedwater heaters operating in parallel.

- Test case 3: A high pressure feedwater system.

The cost of sensors of various types and the values of fault diagnoses are listed in Table 2, as obtained from literature review [13]. Approximate costs of pressure, temperature and flow rate sensors were chosen according to the sensor vendor OMEGA [14]. The scaling factors for fault diagnoses in the cost function were set to relatively large values. With these settings, the GA is expected to find a sensor set with the least cost that is able to diagnose as many faults as possible since the scaling factors are set large.

Table 2. Sensor cost and fault diagnosis scaling factor of the second case

\begin{tabular}{|c|c|}
\hline Sensor/fault & Cost/scaling factor \\
\hline Flow meter & 1200 \\
\hline Temperature sensor & 200 \\
\hline Pressure sensor & 1500 \\
\hline Unique diagnosis of a fault & 30000 \\
\hline Multiple diagnoses of a fault* & 15000 \\
\hline
\end{tabular}

*PRO-AID may find multiple diagnoses which are all mathematically correct. It indicates that the given sensor set could not differentiate between the multiple diagnoses including the actual fault, so a reduced scaling factor is assumed. 


\subsection{Case 1: Single Phase Heat Exchanger}

This test case was developed to validate the algorithm. It is attractive as the optimality of the returned sensor set can be verified manually.

We consider a simple single-phase counterflow heat exchanger, as illustrated by Figure 7. The heat exchanger consists of two sides of fluid flows, a tube side and a shell side. Hot fluid, usually flowing on the tube side, transfers energy to heat up the cold fluid on the shell side. For the test case, the fluid on both sides is assumed to be subcooled liquid water.

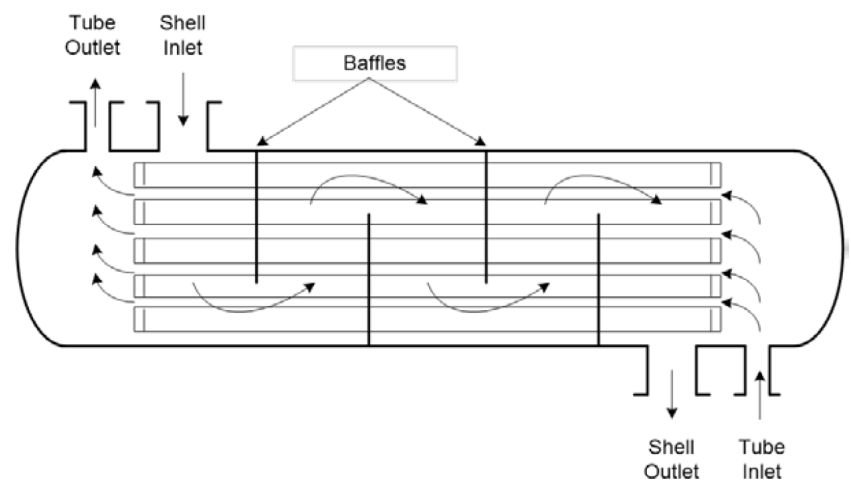

Figure 7. A single-pass shell-tube heat exchanger

For the single-phase flows, relevant T-H variables are mass flowrate, temperature and pressure. One has the option to place a flow meter, a temperature sensor and/or a pressure sensor at each inlet and outlet of each side. In total, there are 12 possible sensors for the two sides.

Beside sensor faults, a list of possible component faults is provided in Table 3.

Table 3. Component faults in the single-phase heat exchanger

\begin{tabular}{|l|l|l|}
\hline Fault ID & Type & Component \\
\hline F1 & Tube leakage & Tube \\
\hline F2 & Tube blockage & Tube \\
\hline F3 & Shell leakage & Shell \\
\hline F4 & Shell blockage & Shell \\
\hline F5 & Fouling & Tube, shell \\
\hline
\end{tabular}

The GA was performed with 10 generations and 100 individuals per generation. As shown in Table 4, the minimized cost starts to converge from Generation 6 . This value is verified by exhausting all sensor combinations in the search space, which consists of $2^{12}=4096$ combinations of the 12 possible sensors. It is found that four combinations of sensors (each with 6 sensors installed) result in the same minimum cost of -71800 . The GA program reported the same value, and the same associated sensor sets. Since the GA program only evaluates the individuals with changed chromosome over a generation, the number of evaluated individuals in each generation is less than the population size (100). The total number of evaluated individuals through the whole calculation is 638, much smaller than the size of the search space (4096). Each evaluation here requires calling PRO-AID for every fault. This number may be further reduced by checking the convergence of the minimum cost and optimize the GA parameters.

The optimal sensor set found in this case consists of six sensors: flowrate, inlet temperature and outlet temperature for each side. This result can also be verified manually based on the underlying physics by analyzing the models that can be constructed. 
Table 4. Results over the GA generations for test case 1

\begin{tabular}{|c|c|c|}
\hline Generation & Minimum cost $^{\mathbf{1}}$ & \# of evaluated individuals $^{\mathbf{2}}$ \\
\hline 1 & -69100 & 65 \\
\hline 2 & -70600 & 40 \\
\hline 3 & -70600 & 59 \\
\hline 4 & -71800 & 66 \\
\hline 5 & -70600 & 46 \\
\hline 6 & -71800 & 48 \\
\hline 7 & -71800 & 61 \\
\hline 8 & -71800 & 58 \\
\hline 9 & -71800 & 46 \\
\hline 10 & -71800 & 49 \\
\hline
\end{tabular}

${ }^{1}$ The true minimum cost is -71800 .

${ }^{2}$ The sum of this column plus the initial 100 individuals' evaluations is the total number of evaluations.

\subsection{Case 2: Two Feedwater Heaters in Parallel}

This test case demonstrates the benefits of the virtual sensor concept in PRO-AID. Given a minimal sensor set, virtual sensors are created automatically in PRO-AID. For this two FWHs case the result is a sensor set (physical + virtual) that provides for the creation of a heat transfer performance model for each FWH. The creation of this model is fundamental to realizing a diagnostic capability for the two FWHs.

This feedwater heater (FWH) test case is a subsystem extracted from a high-pressure feedwater system of a typical PWR plant [5]. It is a single heating stage consisting of two FWHs in parallel piping lines. The structure of the system in consideration is illustrated by the P\&ID in Figure 8. Each FWH is of the vertical channel down shell-tube design. The steam flows in on the shell side, exchanges heat with the feedwater on the tube side and turns into condensate collected in the drain pool.

In the diagnostic problem, we are interested in using sensor readings (flowrate, temperature, and pressure sensors) at various locations in the system to monitor the performance of the two feedwater heaters. Based on physics intuition and practical considerations, several sensors are eliminated from the sensor search space. For example, the inlet flows on the hot side of the FWHs are two-phase mixtures, thus the temperature sensors at those locations are not relevant. Together, the sensor search space includes 34 sensors, i.e., about $1.7 \times 10^{10}$ combinations. For a search space of such size, exhausting all combinations is infeasible.

In setting up the sensor assignment problem, we are interested in detecting fouling, tube leakage/blockage and shell leakage/blockage in each FWH. Additionally, the value of detecting a sensor fault is also accounted for in the cost function. The GA parameters used for this test are listed in Table 5.

Table 5. GA parameters used for test case 2

\begin{tabular}{|c|c|}
\hline Parameter & Value \\
\hline Number of individuals & 1000 \\
\hline Number of generations & 20 \\
\hline Tournament size for selection & 3 \\
\hline Crossover probability & 0.5 \\
\hline Mutation probability & 0.1 \\
\hline Mutation probability to flip a gene & 0.03 \\
\hline
\end{tabular}




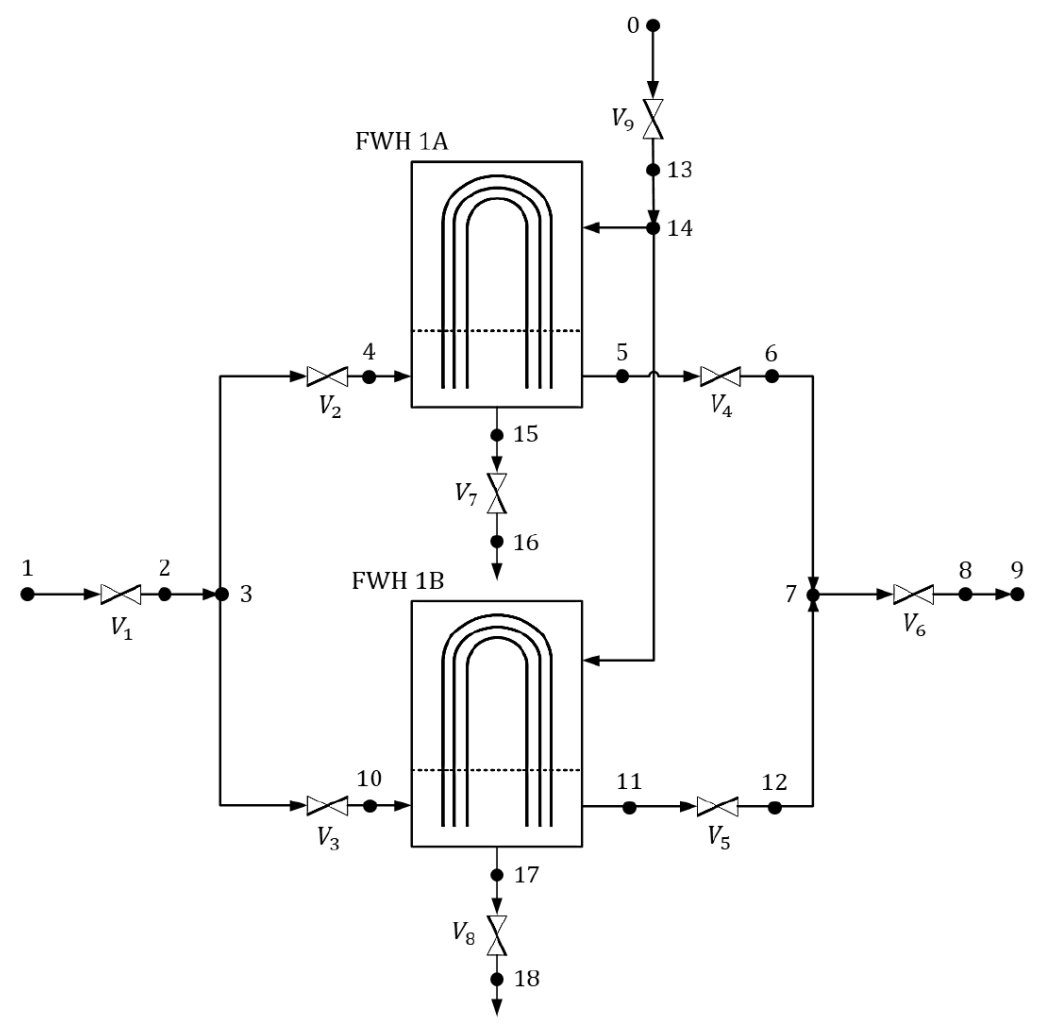

Figure 8. The P\&ID for the system of two FWHs in parallel

To monitor the heat transfer performance of each $\mathrm{FWH}$, the construction of a heat transfer model for a standalone feedwater heater requires a minimum set of six sensors: feedwater flowrate, feedwater inlet temperature, feedwater outlet temperature, drain flowrate and steam inlet pressure. This minimum sensor set is illustrated in the P\&ID in Figure 9, where the 'FE' tag indicates a flowrate sensor, 'TE' a temperature sensor and 'PT' a pressure sensor.

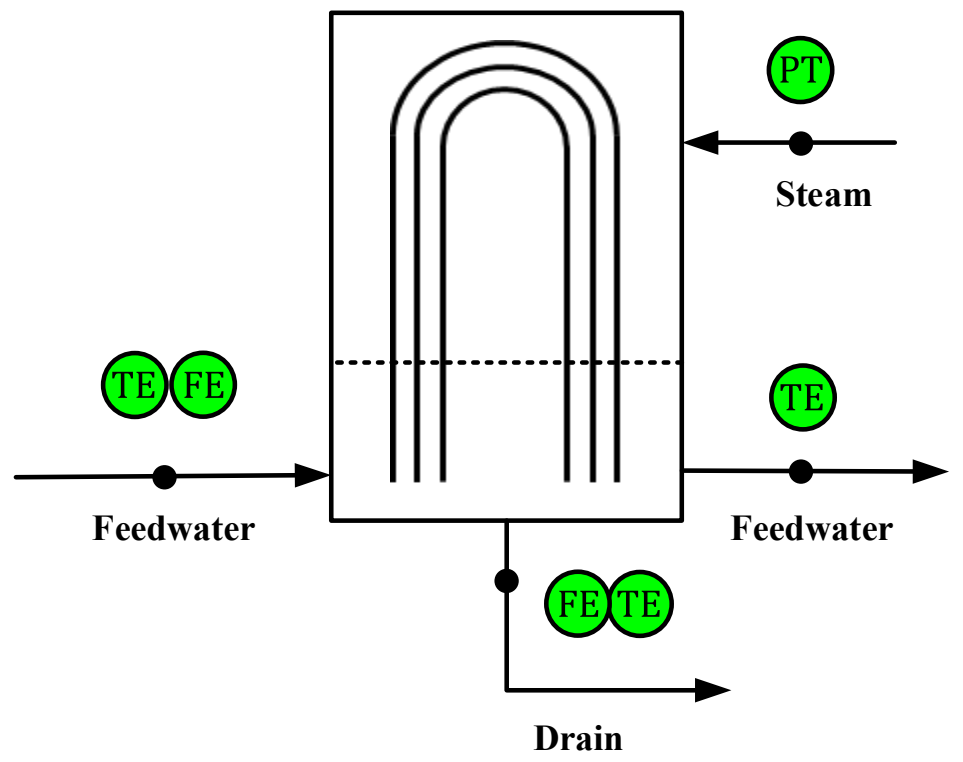

Figure 9. Sensor set requirement for a standalone FWH diagnostic model 
The solution of the GA optimizer yielded multiple optimal sensor-sets all with the same value for the minimized cost functions. From the cost perspective, those sensor sets are equivalent. This is to be expected as different combinations of sensors can allow the detection of the same number of faults. Furthermore, in the current test case, we set the same value for different fault types. One of the optimal sensor sets is listed in Table 6. A summary of the code output for this sensor set is given below in Table 7.

Table 6. An optimal sensor set of test case 2.

\begin{tabular}{|l|l|l|}
\hline ID & Sensor & Description \\
\hline 1 & v6:flow:in & Total feedwater flowrate at the inlet of valve V6 \\
\hline 2 & v9:press:out & Steam pressure at outlet of valve V9 (coming into the two FWH) \\
\hline 3 & v9:flow:out & Total steam flowrate at outlet of valve V9 (coming into the two FWH) \\
\hline 4 & fwh_1a_cold:temp:out & FWH 1A feedwater outlet temperature \\
\hline 5 & fwh_1b_cold:temp:in & FWH 1B feedwater inlet temperature \\
\hline 6 & fwh_1b_cold:temp:out & FWH 1B feedwater outlet temperature \\
\hline 7 & fwh_1a_hot:temp:out & FWH 1A drain outlet temperature \\
\hline 8 & fwh_1b_hot:temp:out & FWH 1B drain outlet temperature \\
\hline 9 & fwh_1b_hot:flow:out & FWH 1B drain outlet flowrate \\
\hline
\end{tabular}

Table 7. Output summary of the selected optimal sensor set for test case 2

\begin{tabular}{|c|}
\hline $\begin{array}{l}\text { Number of available sensors / Number of sensors selected / Minimum cost } \\
\text { 34 / } 9 \text { / -1496550.0 } \\
\text { 7. v6:flow:in } \\
\text { 9. v9:press:out } \\
\text { 12. v9:flow:out } \\
\text { 14. fwh_1a_cold:temp:out } \\
\text { 19. fwh_1b_cold:temp:in } \\
\text { 20. fwh_1b_cold:temp:out } \\
\text { 25. fwh_1a_hot:temp:out } \\
\text { 30. fwh_1b_hot:temp:out } \\
\text { 34. fwh_1b_hot:flow:out } \\
\text {-------Faults uniquely diagnosed (2)------------- } \\
\text { 1. Fouling-fwh_1a_cold } \\
\text { 4. Fouling-fwh_1b_cold } \\
\text {----------Faults non-uniquely diagnosed (15)------------ } \\
\text { 2. Leakage-fwh_1a_cold } \\
\text { 3. Blockage-fwh_1a_cold } \\
\text { 5. Leakage-fwh_1b_cold } \\
\text { 6. Blockage-fwh_1b_cold } \\
\text { 7. Leakage-fwh_1a_hot } \\
\text { 9. Leakage-fwh_1b_hot } \\
\text { 12. SensorFault-fwh_1a_cold:temp:out } \\
\text { 17. SensorFault-fwh_1b_cold:temp:in } \\
\text { 18. SensorFault-fwh_1b_cold:temp:out } \\
\text { 23. SensorFault-fwh_1a_hot:temp:out } \\
\text { 28. SensorFault-fwh_1b_hot:temp:out }\end{array}$ \\
\hline
\end{tabular}




32. SensorFault-fwh_1b_hot:flow:out
39. SensorFault-v6:flow:in
41. SensorFault-v9:press:in
44. SensorFault-v9:flow:out
-------Faults not diagnosed (2)------------
8. Blockage-fwh_1a_hot
10. Blockage-fwh_1b_hot

For clarifications, the nine sensors in this optimal set are represented by the green tags in the P\&ID in Figure 10. Comparing to the minimum six sensors required for each FWH as shown in Figure 9, this sensor set does not meet the requirements for each standalone FWH. Instead, PRO-AID can make use of the balance equations and relations between the FWHs to generate additional virtual sensors. For this case, the resultant virtual sensors are shown by the red tags in Figure 10.

Since the two FWHs share the same feedwater source, the virtual temperature sensor at location 4 (feedwater inlet of FWH 1A) can be created using the temperature reading at location 10. The virtual drain flowrate sensor at 15 can be computed using the two flowrate sensors at 13,17 and the mass balance relation. The two virtual feedwater flowrate sensors at 4 and 5 can be obtained from solving the heat balance equations between the two FWHs. Finally, the pressure sensors at 13 can be used to provide the required pressure reading for both FWHs.

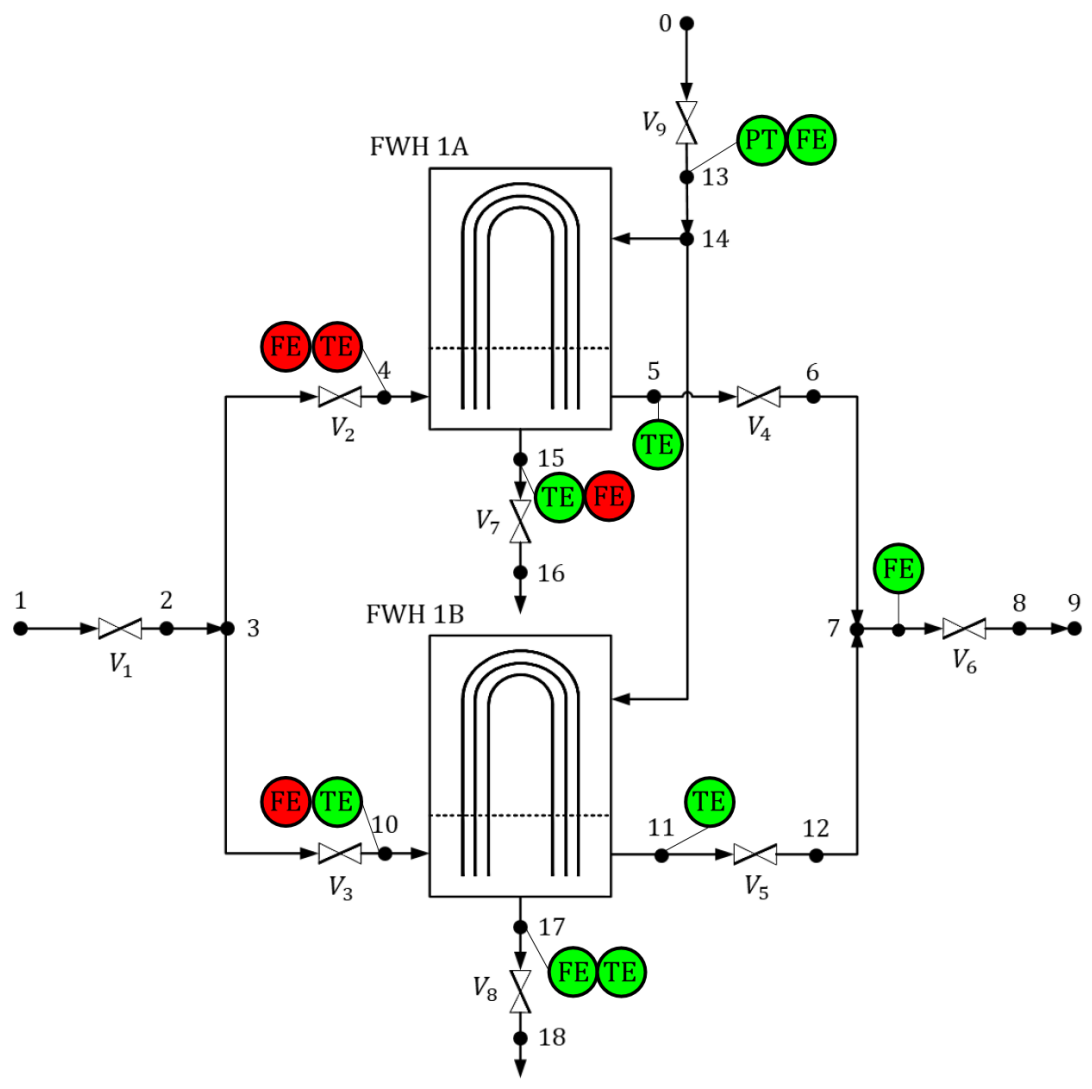

Figure 10. The optimal sensor set (green) and virtual sensors (red) created in PRO-AID for test case 2 
Overall, this set of nine real sensors (green) and four virtual sensors (red) provide a sufficient sensor set for the creation of a heat transfer model for each FWH and also a feedwater flowrate ratio model between the two parallel lines. These models then can be used to detect the faults as showed in the summary in Table 7.

\subsection{Case 3: High Pressure Feedwater System}

This test case demonstrates that compared to the currently installed sensor set, better diagnostic capabilities can be achieved with an optimized sensor set that has fewer sensors.

The high-pressure feedwater system is part of the condensate and feedwater system that is responsible for the supply of pre-heated feedwater to the steam generators. We consider the last two heating stages closest to the inlets of the steam generators known as the first-point and second-point stages. The feedwater heater in each stage is of the closed two-shell type. Each heating stage consists of two feedwater heaters in parallel piping lines. The P\&ID for this system is shown in Figure 11. The system as shown also include three feed pumps and three drain pumps.

From the top of the P\&ID, two-phase mixtures from the high-pressure turbine and moisture separator reheaters flow into the shells of the first and second-point heaters. The first-point heaters drain to the shells of the second-point heaters. On the right of the P\&ID, feedwater from earlier heating stages flows through the two second-point heaters to the suction header of the feed pumps. Drains from the two-point heater shells are collected by the high- pressure heater drain receivers. Drains from the moisture separators (not shown) are collected by a third drain receiver. The three drain pumps pump condensate from the drain receivers to the suction header of the feed pumps. During normal operation, only two of the three feed pumps operate to pump feedwater through the two first- point heaters to a discharge header to supply the steam generators. In emergency situations, excessive drains from the first-point heaters and the drain receivers are collected by a condenser. All the valves to the condenser are otherwise closed off during normal operation.

The yellow tags in the P\&ID in Figure 5 represent the sensor set available in this system. Each label containing PT denotes a pressure transmitter, FE denotes a flowrate sensor and TE denotes a temperature sensor. Let us first review the diagnostic capabilities of this installed sensor set.

First, recall from Figure 9 that the construction of a diagnostic model for a standalone feedwater heater requires at least six measurements: feedwater flowrate, feedwater inlet temperature, feedwater outlet temperature, drain flowrate and steam inlet pressure. The available sensor set for each first-point FWH does not include the required flowrate sensor. However, by utilizing the available sensors and auxiliary equations for the system, a virtual sensor can be obtained. Second, for the second-point FWHs, both the feedwater flowrate and drain flowrate are missing and cannot be obtained from auxiliary equations. It is therefore not possible to construct diagnostic models for the second-point FWHs. Third, for each drain pump, a model can be constructed for the pump head as a function of the flowrate given the available sensors. Fourth, for the feed pumps, no diagnostic models can be constructed. Table 8 lists the diagnostic models that can be generated given the sensor set in Figure 11.

Table 8. Available diagnostic models from the installed sensor set test case 3

\begin{tabular}{|c|l|l|l|l|}
\hline ID & Name & Model Type & Components & Relevant Fault Types \\
\hline 1 & FR-1 & Flow ratio & FWHs 1A and 1B & Leakage, Blockage \\
\hline 2 & FWH-1A & HX performance & FWH 1A & Leakage, Fouling \\
\hline 3 & FWH-1B & HX performance & FWH 1B & Leakage, Fouling \\
\hline 4 & SDP-1A & Pump head & Drain pump 1A & Pump fault \\
\hline 5 & SDP-1B & Pump head & Drain pump 1B & Pump fault \\
\hline 6 & SDP-1C & Pump head & Drain pump 1C & Pump fault \\
\hline
\end{tabular}


Using these models, the list of component faults that can be detected are listed in Table 9. However, none of these faults can be uniquely diagnosed, i.e., the diagnosis for each case includes additional possibilities of other component faults or sensor faults. In particular, with the installed sensor set, we cannot detect or diagnose any faults in the feedwater pumps or the second-point FWHs.

Table 9. Detectable component faults using the installed sensor set for test case 3

\begin{tabular}{|c|c|c|c|c|}
\hline Fault ID & Comp. Tag & Comp. Name & Comp. Type & Fault \\
\hline 1 & 1-FW-E-1A & FWH 1A & FWH & Fouling \\
\hline 2 & 1-FW-E-1A & FWH 1A & FWH & Tube leak \\
\hline 3 & 1-FW-E-1A & FWH 1A & FWH & Shell leak \\
\hline 4 & 1-FW-E-1A & FWH 1A & FWH & Tube block \\
\hline 5 & 1 -FW-E-1B & FWH 1B & FWH & Fouling \\
\hline 6 & 1 -FW-E-1B & FWH 1B & FWH & Tube leak \\
\hline 7 & 1-FW-E-1B & FWH 1B & FWH & Shell leak \\
\hline 8 & 1-FW-E-1B & FWH 1B & FWH & Tube block \\
\hline 9 & 1-SD-P-1A & SDP 1A & Pump & Pump fault \\
\hline 10 & 1-SD-P-1B & SDP 1B & Pump & Pump fault \\
\hline 11 & 1-SD-P-1C & SDP 1C & Pump & Pump fault \\
\hline
\end{tabular}




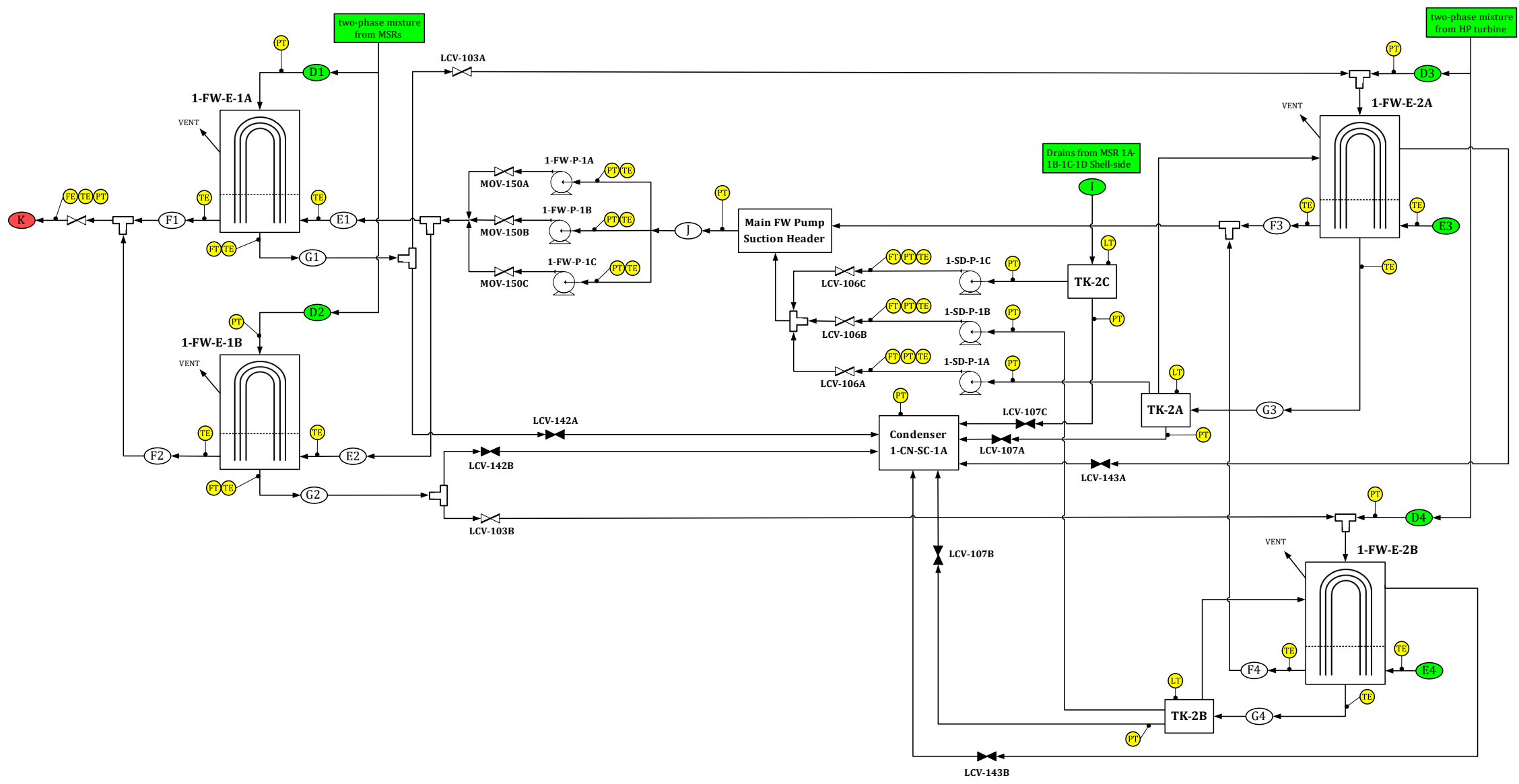

Figure 11. The P\&ID with the installed sensor set for the feedwater system in test case 3 


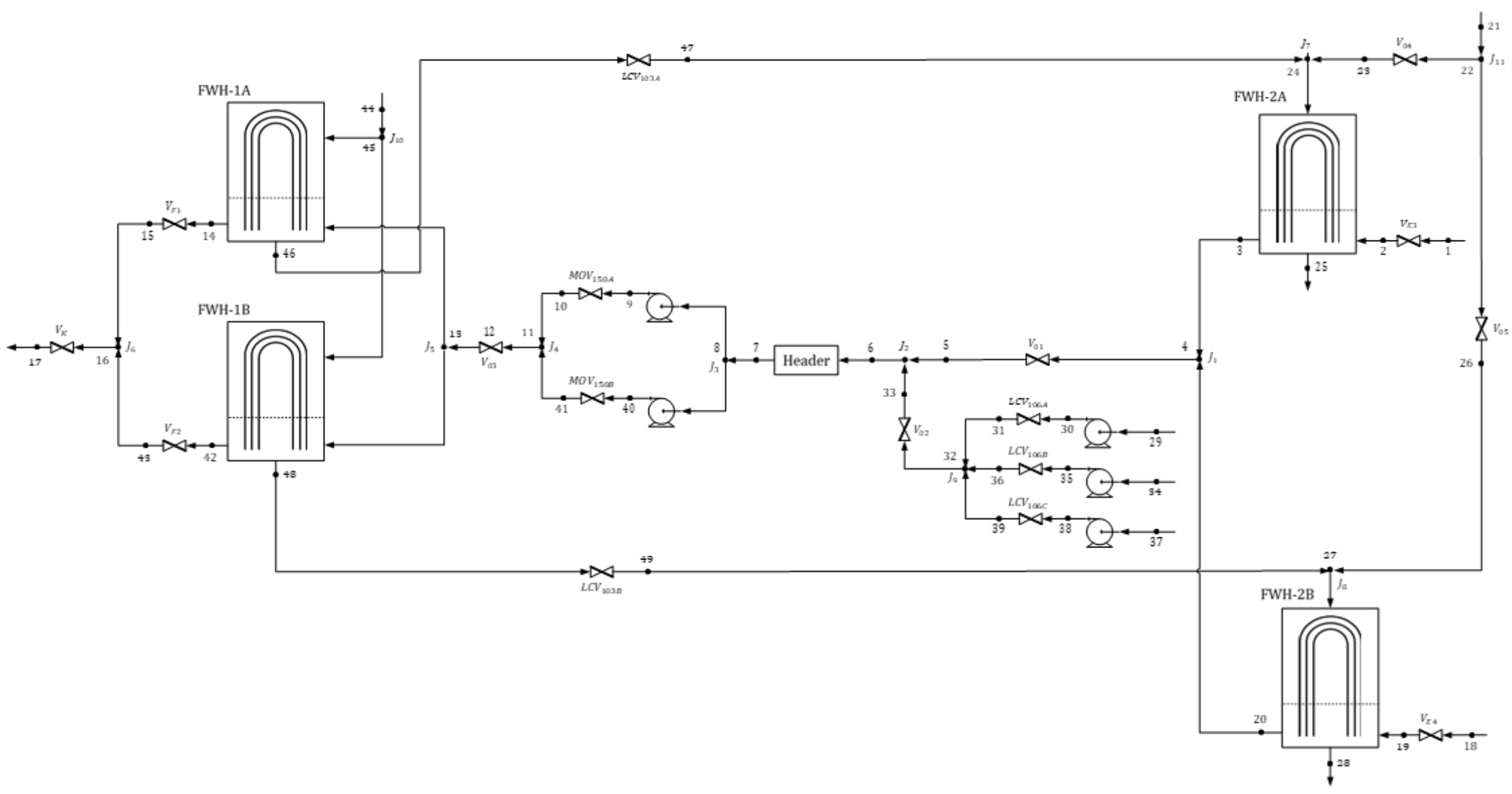

Figure 12. Simplified P\&ID used in PRO-AID for test case 3 
For a demonstration of the sensor assignment problem, let us assume that the objective is to detect the main faults in every key component of the system, i.e. fouling, tube leakage/blockage, shell leakage in each FWH; pump, bearings and motor faults in each feed pump; and pump fault in each drain pump. A complete list of the faults is given below in Table 10. Again, the two numbers for each fault indicate the scaling factors for a unique diagnosis and multiple-fault diagnosis of the fault.

Table 10. Faults of interest in Test Case 3

\begin{tabular}{|c|c|c|c|c|}
\hline FAULT & Fouling & fwh_1a & 300000 & 150000 \\
\hline FAULT & Fouling & fwh_1b & 300000 & 150000 \\
\hline FAULT & Fouling & $\mathrm{fwh} \_2 \mathrm{a}$ & 300000 & 150000 \\
\hline FAULT & Fouling & $\mathrm{fwh} \_2 \mathrm{~b}$ & 300000 & 150000 \\
\hline FAULT & Leakage & fwh_1a_cold & 300000 & 150000 \\
\hline FAULT & Blockage & fwh_1a_cold & 300000 & 150000 \\
\hline FAULT & Leakage & fwh_1b_cold & 300000 & 150000 \\
\hline FAULT & Blockage & fwh_1b_cold & 300000 & 150000 \\
\hline FAULT & Leakage & fwh_1a_hot & 300000 & 150000 \\
\hline FAULT & Leakage & fwh_1b_hot & 300000 & 150000 \\
\hline FAULT & Leakage & fwh_2a_cold & 300000 & 150000 \\
\hline FAULT & Blockage & fwh_2a_cold & 300000 & 150000 \\
\hline FAULT & Leakage & fwh_2b_cold & 300000 & 150000 \\
\hline FAULT & Blockage & fwh_2b_cold & 300000 & 150000 \\
\hline FAULT & Leakage & fwh_2a_hot & 300000 & 150000 \\
\hline FAULT & Leakage & fwh_2b_hot & 300000 & 150000 \\
\hline FAULT & Pump & fwp_1a & 300000 & 150000 \\
\hline FAULT & Motor & fwp_1a & 300000 & 150000 \\
\hline FAULT & Bearings & fwp_1a & 300000 & 150000 \\
\hline FAULT & Pump & fwp_1b & 300000 & 150000 \\
\hline FAULT & Motor & fwp_1b & 300000 & 150000 \\
\hline FAULT & Bearings & fwp_1b & 300000 & 150000 \\
\hline FAULT & Pump & sdp_1a & 300000 & 150000 \\
\hline FAULT & Pump & sdp_1b & 300000 & 150000 \\
\hline FAULT & Pump & $\mathrm{sdp} \_1 \mathrm{c}$ & 300000 & 150000 \\
\hline
\end{tabular}

The GA parameters used for this test are listed in Table 11. Possible sensor spawning locations include the inlet and outlet ports of all the components shown in the P\&ID in Figure 11. However, to reduce runtime, we do not consider all possible sensors but instead narrow the list by removing some sensors at certain locations that would be considered unreasonable from a practical perspective. For example, if we are only interested in the key components with the faults listed in Table 10, it is not necessary to have pressure sensors at both the inlet and outlet of a valve, nor temperature sensors at both the inlet and outlet of a pump. In total, the search space for the optimization problem includes 59 sensors. 
Table 11. GA parameters used for test case 3

\begin{tabular}{|c|c|}
\hline Parameter & Value \\
\hline Number of individuals & 2000 \\
\hline Number of generations & 20 \\
\hline Tournament size for selection & 3 \\
\hline Crossover probability & 0.5 \\
\hline Mutation probability & 0.1 \\
\hline Mutation probability to flip a gene & 0.03 \\
\hline
\end{tabular}

In this case, the optimizer found a unique optimal set of 38 sensors that would yield the lowest cost of -4311600.0 . A summary of the result is given below in Table 12, which lists the selected sensors and the faults that can be uniquely diagnosed, non-uniquely diagnosed and cannot be detected.

Table 12. Summary of the optimal sensor set for test case 3

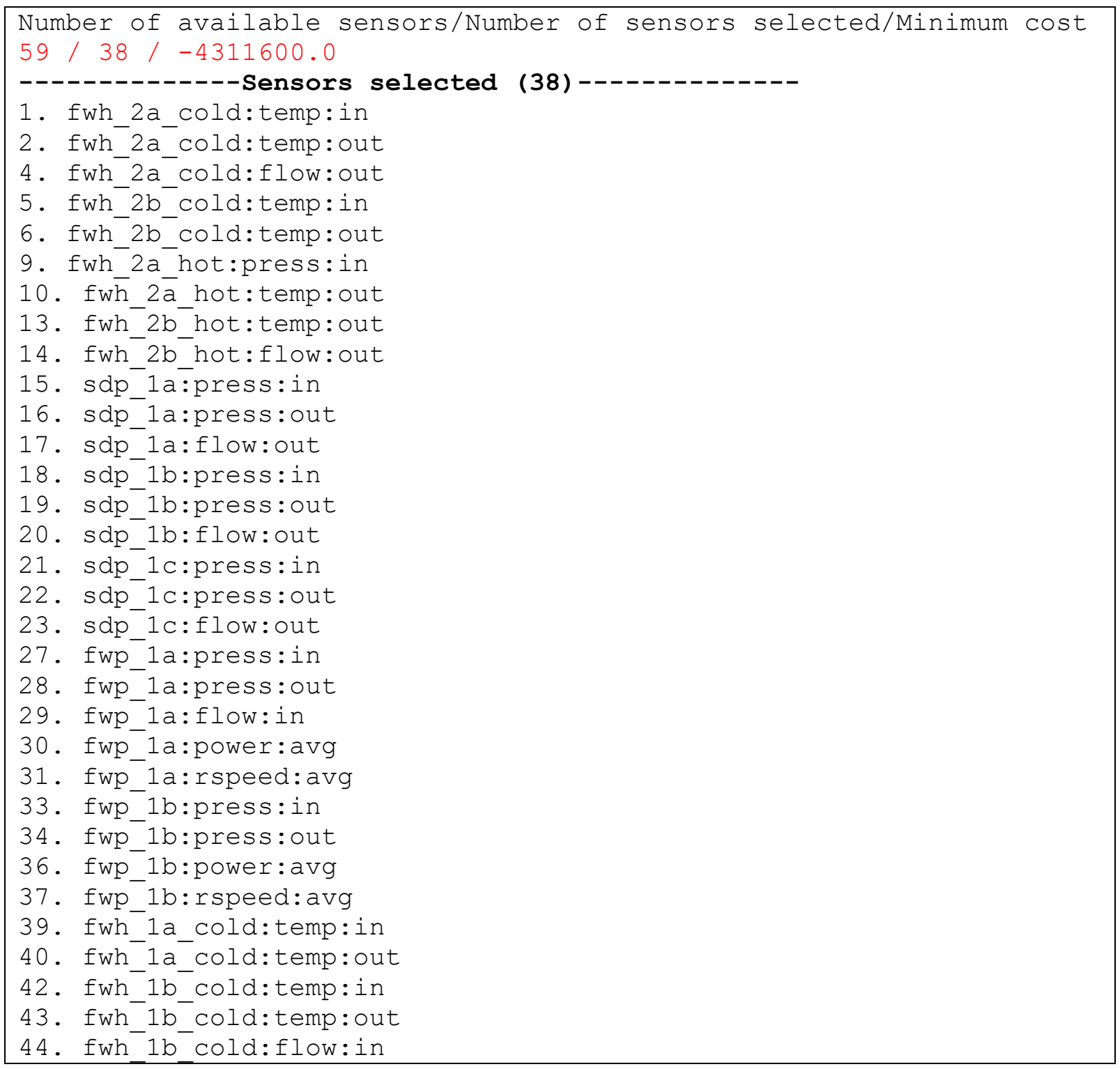




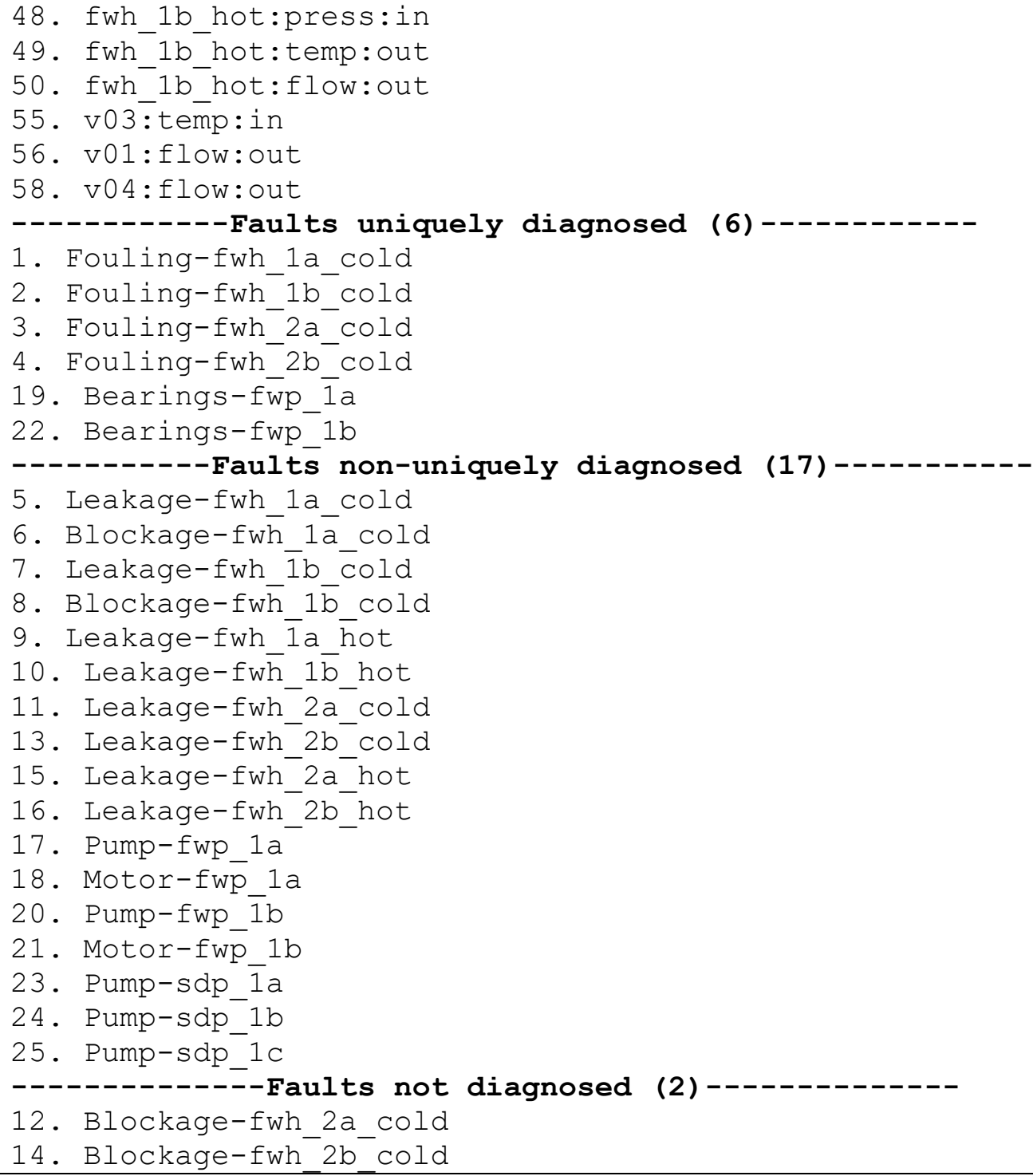

Given this sensor set, the list of diagnostic models that can be constructed in PRO-AID is shown in Table 13

Table 13. PRO-AID diagnostic models given the optimal sensor set for Test Case 3

\begin{tabular}{|c|l|l|l|l|}
\hline ID & Name & Model Type & Components & Relevant Fault Types \\
\hline 1 & FR-1 & Flow ratio & FWHs 1A and 1B & Leakage, Blockage \\
\hline 2 & FWH-1A & HX performance & FWH 1A & Leakage, Fouling \\
\hline 3 & FWH-1B & HX performance & FWH 1B & Leakage, Fouling \\
\hline 4 & FWH-2A & HX performance & FWH 2A & Leakage, Fouling \\
\hline 5 & FWH-2B & HX performance & FWH 2B & Leakage, Fouling \\
\hline 6 & SDP-1A & Pump head & Drain pump 1A & Pump fault \\
\hline 7 & SDP-1B & Pump head & Drain pump 1B & Pump fault \\
\hline 8 & SDP-1C & Pump head & Drain pump 1C & Pump fault \\
\hline 9 & FWP-1A-H & Pump head & FWP 1A & Pump/Bearings/Motor \\
\hline 10 & FWP-1A-M & Motor power & FWP 1A & Pump/Bearings/Motor \\
\hline
\end{tabular}




\begin{tabular}{|l|l|l|l|l|}
\hline 11 & FWP-1A-O & $\begin{array}{l}\text { Overall pump and } \\
\text { motor performance }\end{array}$ & FWP 1A & Pump/Bearings/Motor \\
\hline 12 & FWP-1B-H & Pump head & FWP 1A & Pump/Bearings/Motor \\
\hline 13 & FWP-1B-M & Motor power & FWP 1A & Pump/Bearings/Motor \\
\hline 14 & FWP-1B-O & $\begin{array}{l}\text { Overall pump and } \\
\text { motor performance }\end{array}$ & FWP 1A & Pump/Bearings/Motor \\
\hline 15 & PR-1 & Parity relation & $\begin{array}{l}\text { FWHs 1A and 1B } \\
\text { inlet temperature } \\
\text { sensors }\end{array}$ & Sensor faults \\
\hline
\end{tabular}

In particular, notice from Table 10 that in this test we are interested in detecting all pump, bearings and motor faults in each feed pump but only the pump fault in each drain pump. As a result, the sensor set shown in Table 12 includes motor power, motor current, and shaft speed for both feed pump in addition to the set of inlet pressure, outlet pressure and flowrate required for all pumps. The list of models in Table 12 includes three models for each feed pump (pump head, motor power and combined power models) in comparison to a single pump head model for each drain pump.

Table 14. Comparisons of diagnostic capabilities of the installed sensor set and the optimal sensor set

\begin{tabular}{|c|c|c|c|c|c|c|}
\hline \multirow{2}{*}{ ID } & \multicolumn{2}{|c|}{ Fault } & \multicolumn{2}{|c|}{$\begin{array}{l}\text { Installed Set } \\
\text { (47 sensors) }\end{array}$} & \multicolumn{2}{|c|}{$\begin{array}{l}\text { Optimal Set } \\
\text { (38 sensors) }\end{array}$} \\
\hline & Comp. & Type & Detect & $\begin{array}{l}\text { Uniquely } \\
\text { Diagnose }\end{array}$ & Detect & $\begin{array}{l}\text { Uniquely } \\
\text { Diagnose }\end{array}$ \\
\hline 1 & FWH $1 \mathrm{~A}$ & Fouling & $\mathrm{Y}$ & $\mathrm{N}$ & $\mathrm{Y}$ & $\mathrm{Y}$ \\
\hline 2 & FWH $1 \mathrm{~A}$ & Tube Leak & $\mathrm{Y}$ & $\mathrm{N}$ & $\mathrm{Y}$ & $\mathrm{N}$ \\
\hline 3 & FWH 1A & Tube Block & $\mathrm{Y}$ & $\mathrm{N}$ & $\mathrm{Y}$ & $\mathrm{N}$ \\
\hline 4 & FWH $1 \mathrm{~A}$ & Shell Leak & Y & $\mathrm{N}$ & Y & $\mathrm{N}$ \\
\hline 5 & FWH 1B & Fouling & $\mathrm{Y}$ & $\mathrm{N}$ & $\mathrm{Y}$ & $\mathrm{Y}$ \\
\hline 6 & FWH 1B & Tube Leak & $\mathrm{Y}$ & $\mathrm{N}$ & $\mathrm{Y}$ & $\mathrm{N}$ \\
\hline 7 & FWH 1B & Tube Block & $\mathrm{Y}$ & $\mathrm{N}$ & $\mathrm{Y}$ & $\mathrm{N}$ \\
\hline 8 & FWH 1B & Shell Leak & $\mathrm{Y}$ & $\mathrm{N}$ & $\mathrm{Y}$ & $\mathrm{N}$ \\
\hline 9 & FWH 2A & Fouling & $\mathrm{N}$ & $\mathrm{N}$ & $\mathrm{Y}$ & $\mathrm{Y}$ \\
\hline 10 & FWH 2A & Tube Leak & $\mathrm{N}$ & $\mathrm{N}$ & $\mathrm{Y}$ & $\mathrm{N}$ \\
\hline 11 & FWH 2A & Tube Block & $\mathrm{N}$ & $\mathrm{N}$ & $\mathrm{N}$ & $\mathrm{N}$ \\
\hline 12 & FWH 2A & Shell Leak & $\mathrm{N}$ & $\mathrm{N}$ & $Y$ & $\mathrm{~N}$ \\
\hline 13 & FWH 2B & Fouling & $\mathrm{N}$ & $\mathrm{N}$ & $Y$ & $\mathrm{Y}$ \\
\hline 14 & FWH 2B & Tube Leak & $\mathrm{N}$ & $\mathrm{N}$ & $\mathrm{Y}$ & $\mathrm{N}$ \\
\hline 15 & FWH 2B & Tube Block & $\mathrm{N}$ & $\mathrm{N}$ & $\mathrm{N}$ & $\mathrm{N}$ \\
\hline 16 & FWH 2B & Shell Leak & $\mathrm{N}$ & $\mathrm{N}$ & $\mathrm{Y}$ & $\mathrm{N}$ \\
\hline 17 & FWP 1A & Pump & $\mathrm{N}$ & $\mathrm{N}$ & $\mathrm{Y}$ & $\mathrm{N}$ \\
\hline 18 & FWP 1A & Motor & $\mathrm{N}$ & $\mathrm{N}$ & $\mathrm{Y}$ & $\mathrm{N}$ \\
\hline 19 & FWP 1A & Bearings & $\mathrm{N}$ & $\mathrm{N}$ & $\mathrm{Y}$ & $\mathrm{Y}$ \\
\hline 20 & FWP 1B & Pump & $\mathrm{N}$ & $\mathrm{N}$ & $Y$ & $\mathrm{~N}$ \\
\hline 21 & FWP 1B & Motor & $\mathrm{N}$ & $\mathrm{N}$ & $\mathrm{Y}$ & $\mathrm{N}$ \\
\hline 22 & FWP 1B & Bearings & $\mathrm{N}$ & $\mathrm{N}$ & $\mathrm{Y}$ & $\mathrm{Y}$ \\
\hline 23 & SDP 1A & Pump & $\mathrm{Y}$ & $\mathrm{N}$ & $\mathrm{Y}$ & $\mathrm{N}$ \\
\hline 24 & SDP 1B & Pump & $\mathrm{Y}$ & $\mathrm{N}$ & $\mathrm{Y}$ & $\mathrm{N}$ \\
\hline 25 & SDP $1 C$ & Pump & $\mathrm{Y}$ & $\mathrm{N}$ & $\mathrm{Y}$ & $\mathrm{N}$ \\
\hline
\end{tabular}


Table 14 provides comparisons between the installed sensor set and the optimal sensor set. As shown in Figure 11, the installed sensor set consists of 47 sensors. However, not all of the installed sensors have direct use for the current diagnostic current. For example, the temperature sensor at the inlet of each feed pump is not relevant if we are only interested in monitoring the hydraulic performance. Nonetheless, those sensors may have been installed for other monitoring needs in the system. The optimal sensor set, on the other hand, consists of 38 sensors that can be used by PRO-AID to create diagnostic models.

It is clear that the optimal sensor set can provide improved diagnostic capabilities over the installed sensor set. Here in Table 14, a "unique diagnosis" refers to the scenario where there is only a single possibility in the diagnostic result. There are cases in which the diagnostic result consists of a unique single-fault diagnosis along with other multiple-fault possibilities. Such cases do not meet the above definition of a "unique diagnosis", but they can be considered to be more informative than scenarios where there are multiple single-fault diagnoses. For instance, with the optimal sensor set, a pump or motor fault in each feed pump will be detected as a unique single-fault event besides other multiple-fault diagnosis. This is to emphasize that Table 14 does not fully summarize the benefits of the optimal sensor set over the installed set.

\subsection{Parallel Efficiency}

To test the parallelization, we ran the first problem with 1000 individuals per generation and 3 generations in total on a Linux multi-processor system. The environment variable OMP_NUM_THREADS is set to 1 to make sure PRO-AID is run in serial (no interference with the parallel testing for the GA). As shown in Figure 13, both multiprocessing and SCOOP shows good speedups up to 16 processors. In general, with more processors, the number of individuals to be evaluated for each processor is smaller, resulting in a larger variation of the PRO-AID running times across the processors, leading to the slight degradation of parallel efficiency.

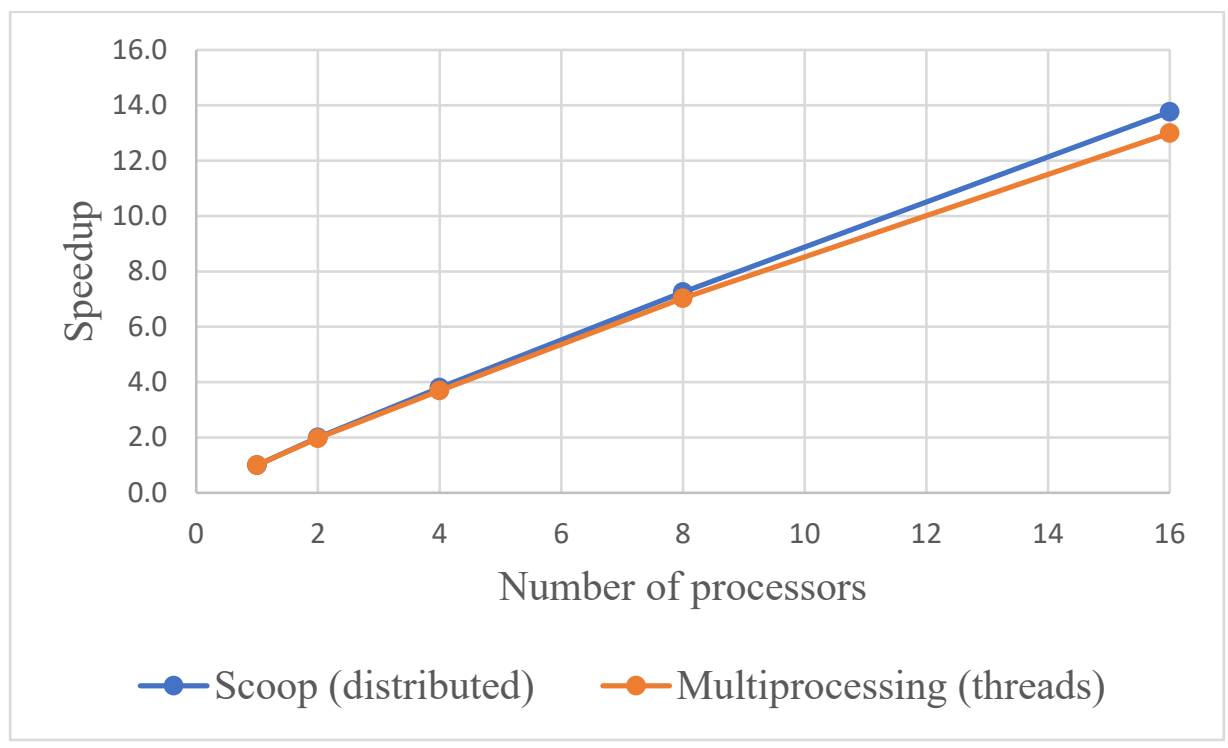

Figure 13 Speed up vs number of processors

A comparison of the computational cost for the three test cases is listed in Table 15.

Table 15. Total runtime of the three test cases.

\begin{tabular}{|l|l|l|}
\hline Test Case & Resources & Runtime \\
\hline 1 & 16 cores at $3.0 \mathrm{GHz}$ & $30 \mathrm{~s}$ \\
\hline
\end{tabular}




\begin{tabular}{|l|l|l|}
\hline 2 & 16 cores at $3.0 \mathrm{GHz}$ & $30 \mathrm{~m}$ \\
\hline 3 & 30 cores at $2.1 \mathrm{GHz}$ & $3 \mathrm{~h} 45 \mathrm{~m}$ \\
\hline
\end{tabular}

\section{DISCUSSIONS}

Based on the fault diagnosis tool PRO-AID, a parallel capability for determining the optimal sensor set is developed. To avoid calculating the derivatives of the cost function, the genetic algorithm is used in this work. A Python program is developed to drive the optimization. It reads the modified PRO-AID and P\&ID inputs to obtain the sensor search space as well as the sensor cost data. The Distributed Evolutionary Algorithms in Python (DEAP) package is used to perform the GA. Parallelization is realized behind the scenes through the built-in interfaces of DEAP to distribute the work of individual evaluations. The initial testing of a single-phase heat exchanger problem and a two-point feedwater heater problem show that the GA program can obtain optimized candidate sensor sets with a few orders less computational efforts as compared to the brutal-force search. When running in parallel, good speedups up to 30 processors was observed for the test problems.

Results of test case 1, whose optimal sensor sets can be verified manually, served to validate the algorithm. Results of test case 2 demonstrated the benefits of the concepts of virtual sensors in PRO-AID. In this case, given a minimal sensor set, various virtual sensors were created for the two FWHs in order to provide a sufficient sensor set for the creation of a heat transfer performance model for each FWH.

In test case 3 with a high-pressure feedwater system, compared to the currently installed sensor set, better diagnostic capabilities can be achieved with an optimized sensor set, even with fewer sensors. It should be noted that some of the sensors in the installed set though cannot be utilized for our current diagnostic approach may have been installed for other monitoring needs. The take-away is that compared to the installed sensor set there exists a more strategic assignment of sensors that will furnish better health monitoring capability and with fewer sensors.

The high-pressure feedwater problem as posed above illustrates the added value of approaching the sensor selection problem as one amenable to algorithmic solution. This problem is of interest to advanced reactor designers and to utilities that are setting up remote monitoring and diagnostic centers. In these test cases, we have only demonstrated the sensor assignment problem using a relatively simple cost function. Better and more diversified results can be obtained by improving the form of the cost function to account for practical considerations, e.g., the expected occurrence frequency and significance of each type of faults, the installation cost of each sensor based on its location, etc.

The results in this report demonstrated the successful use of the GA for sensor assignment optimization. Optimal results with physically explainable sensor sets were obtained in reasonable runtime. A parametric study of the other GA parameters such as the number of generations, crossover and mutation probabilities to further improve the convergence speed is an ongoing work. Also, the study of combining the GA with other techniques to obtain a guaranteed global optimum is another future work. 


\section{APPENDIX A - PRO-AID INPUT AND OUTPUT FILES}

\section{A.1 PRO-AID Main Input File}

The main input file is used to specify the location of the P\&ID input file, a list of faults for a scenario to be considered and user options for the reasoning process. A sample input file is given below:

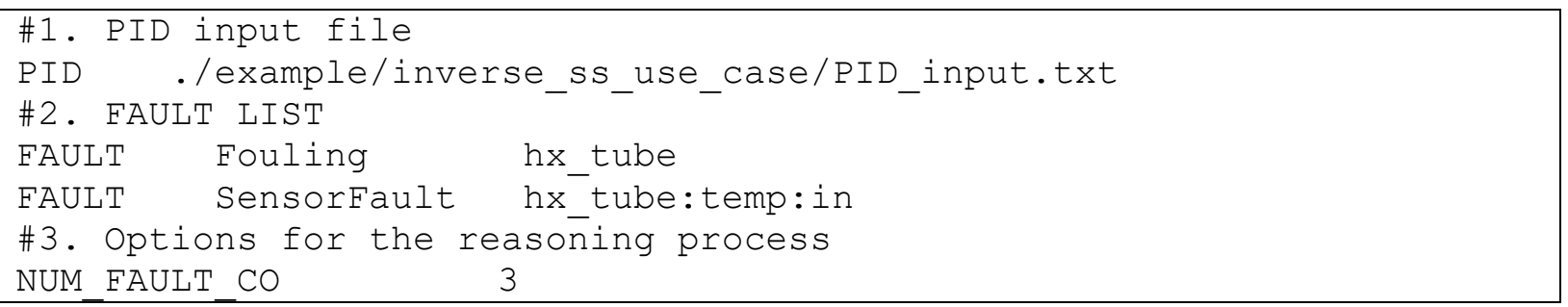

Each line of the input file is recognized by a keyword. Lines starting with \# are treated as comment lines and will be ignored.

Location of the P\&ID input file is provided by a line with the keyword "PID" following by the path to the file.

For the fault list, each line represents a fault in the format of:

FAULT $\quad$ [Fault type $] \quad$ [Component name $]$

Valid component fault types include: Fouling, Leakage, Blockage. Sensor fault type is denoted by SensorFault.

Component names must match those in the P\&ID input file. In PRO-AID, sensors at the inlet or outlet of each component are named by default in a format specifying its type and location with respect to the 'parent' component: "component_name:sensor_type:location". For example, hx_tube:temp: in refers to the temperature sensor at the inlet of the component named hx_tube (tube side of a heat exchanger).

Valid sensor types include: temp for temperature, press for pressure, flow for flowrate.

Valid sensor locations (w.r.t a component): in for inlet and out for outlet.

In the third section, NUM_FAULT_CO sets the cutoff limit for number of faults per diagnosis. Its exact meaning will be explained in the output file. This input is optional, by default, NUM_FAULT_CO is set to 3.

\section{A.2 P\&ID Input File}

The P\&ID is imported via an input text file using a format inherited from the previous version of qualitative PRO-AID. The input file consists of 5 sections, some of which are not relevant to the quantitative approach and currently unused. Details of the main sections are described below.

Section 1a: Component list

Each single inlet/outlet component is assigned a unique outlet index. Each line in this section represents a component and should consist of:

[outlet index], [component name], [component type] 
Each heat exchanger is separated into two components, one for each side. Each junction (with multiple inlet or outlet flows) only needs to be declared once, with the outlet index now understood to be at the "center" of the junction.

Example:

\begin{tabular}{|l|}
\hline 1, hot_inlet, system, \\
2, hx_tube, counterflow_hx_tube, \\
3, cold_inlet, system, \\
4, hx_shell, counterflow_hx_shell,
\end{tabular}

Section 1e: Adjacencies

Since each heat exchanger was separated into two single inlet/outlet components, we need to keep track of which two components belong to the same heat exchanger. The two sides of a HX are defined to be "adjacent" to each other. Information on adjacency is listed in this section. For example, with two sides of a heat exchanger:

hx_tube, hx_shell

Section 3: Sensors

Each line lists all the sensors available to each component:

[index], [component name], [sensor1], [sensor2], ..., [sensorN],

The first item (index) is not relevant. Each sensor is provided in a short-handed format: e.g. ' $t i$ ' for inlet temperature; 'po' for outlet pressure, etc.

Example:

1, hx_tube, fi, ti, to

2, hx_shell, fi, ti, to

Here, the first line specifies an inlet flowrate, inlet temperature and outlet temperature sensors available to the tube side of the heat exchanger.

Section 5a: Loops and their Components

Each line in this section represents a mono-directional flow between two end points which is called a loop. Each line should consist of a name for the loop followed by a series of outlet indices for its components:

$$
\text { loop_1, 1, 2, }
$$

Each loop should start and end at either an end point (at the boundary of the system) or at a junction but can contain junctions among its list of components.

\section{A.3 PRO-AID Output File}

The output file summarizes the diagnostic result as determined by PRO-AID for the fault scenario in consideration. Depending on the available sensor set, the diagnostic result may consist of a unique diagnosis or multiple diagnoses. 
Each diagnosis is denoted by the square brackets "[]" containing a list of faults. For instance, $\left[F_{0}, F_{3}\right]$ is a diagnosis claiming both faults $F_{0}$ and $F_{3}$ are present. Faults are written in the output file in the format of "FaultType-ComponentName".

For example, [Fouling-hx_shell, Sensorfault-hx_shell:flow: in] is a diagnosis claiming there is Fouling in the heat exchanger and at the same time a Sensor Fault in the flowrate sensor at the inlet of the shell side.

In general, the diagnostic output may contain multiple diagnoses, all of which are mathematically valid for the fault scenario in consideration. That is the case when, given the available sensor set, PRO-AID cannot differentiate between the multiple possibilities.

Intuitively, diagnoses with more faults are usually less likely to be true. However, that is not always true depending on the prior probabilities of the faults involved. Nonetheless, for the current problem, one can choose to ignore diagnoses with too many faults by setting a cutoff limit on the number of faults per diagnosis using the "NUM_FAULT_CO" option in the input file. By default, the limit is set to 3 and PROAID will only output diagnoses with 3 or fewer faults. 


\section{APPENDIX B - DESCRIPTION OF THE GA INPUT PARAMETERS}

\section{B.1 Input Description}

The inputs of the program include modified PRO-AID and P\&ID inputs with the costs of sensors, scaling factors of fault diagnoses and optimization options.

\section{B.2 Modified PRO-AID Input}

Two places in the original PRO-AID input are modified for running the optimization.

The first place is to include the 'gain' (scaling factor) for successfully diagnosing a fault. Currently, two values should be added after each fault definition where the first one is for the fault to be uniquely diagnosed, and the second one is when multiple diagnoses coexist including the correct one, e.g.,

\begin{tabular}{|lllll|}
\hline FAULT & Fouling & hx_shell & 30000 & 15000 \\
FAULT & Leakage & hx_tube & 20000 & 10000 \\
\hline
\end{tabular}

The second place is to add the optimization options at the end of the input file. The following parameters (case sensitive) may be specified,

\begin{tabular}{|c|c|c|c|c|}
\hline Parameter & Description & Type & $\begin{array}{l}\text { Recommended } \\
\text { Range }\end{array}$ & Default \\
\hline NUM INDIVIDUALS & Number of individuals & Integer & $100-2000$ & 100 \\
\hline NUM_GENERATIONS & Number of generations & Integer & $10-20$ & 10 \\
\hline CROSSOVER_PROB & Crossover percentage on population & Float & $0.5-1.0$ & 0.5 \\
\hline MUTATE_PROB & $\begin{array}{l}\text { Mutation percentage on population } \\
\text { and probabilities to flip genes }\end{array}$ & $\begin{array}{l}\text { Two } \\
\text { floats }\end{array}$ & $0.1-0.2 \quad 0.01-0.1$ & 0.20 .5 \\
\hline SELECT_TOURNSIZE & Tournament size for selection & Integer & $3-5$ & 3 \\
\hline NUM_BĒST & $\begin{array}{l}\text { Number of best candidates edit in } \\
\text { output }\end{array}$ & Integer & $\leq$ NUM_INDIVIDUALS & 10 \\
\hline NUM_PROC & Number of processors (threading) & Integer & None & 1 \\
\hline RANDOM_SEED & $\begin{array}{l}\text { The seed for random number } \\
\text { generation }\end{array}$ & Integer & None & 64 \\
\hline
\end{tabular}

Some notes for setting up the parameters:

1. The number of individuals is per generation.

2. Two-point crossover is used for the crossover operation (the bits in between the two random points are swapped between the parents).

3. The first value for MUTATE_PROB determines the percentage of population to be mutated, then each gene of these individuals use the second value to determine whether to flip the binary.

4. For the tournament selection, 'tournaments' will be run among a few individuals chosen at random from the population, and the one with best fitness is selected for crossover. With larger tournament size, weak individuals have a smaller chance to be selected.

5. Changing the random seed will result in slightly different optimum sensor set, unless the number of individuals is sufficiently large. 
An example of specifying these options:

\begin{tabular}{|l|l|}
\hline \# 4. Parameters for the optimization problem \\
NUM_INDIVIDUALS 400 \\
NUM_GENERATIONS 20 \\
CROSSOVER_PROB 0.5 \\
MUTATE_PROB 0.10 .03 \\
SELECT_TOURNSIZE 3 \\
NUM_BEST 400 \\
NUM_PROC 25 \\
RANDOM_SEED 1
\end{tabular}

The default values in the table above will be used if a card/value is omitted.

\section{B.3 Modified P\&ID Inputs}

Only the 'Sensors' block in the P\&ID input needs to be modified to include all candidate sensors of each component as the search space and their cost data. The cost data are provided after sensor specifications. The first line is the procuring cost for each sensor, and the second line is the installation cost, e.g.,

1. hx_tube, fi, ti, to, pi, po, fo

$1200.0,200.0,200.0,1500.0,1500.0,1200.0$

$0.0,0.0,0.0,0.0,0.0,0.0$ 


\section{REFERENCES}

[1] R. Vilim, T. N. Nguyen and R. Ponciroli, "Explainable and Trustworthy Diagnostics Achievable through Process-Based Automated Reasoning," in 12th Nuclear Plant Instrumentation, Control and Human-Machine Interface Technologies, Virtual Meeting, June 14-17, 2021.

[2] R. Vilim and T. N. Nguyen, "Physics-based Automated Reasoning for Health Monitoring: Sensor Set Selection," in 12th Nuclear Plant Instrumentation, Control and Human-Machine Interface Technologies, Virtual Meeting, June 14-17, 2021.

[3] R. Vilim, T. Nguyen, H. Wang and R. Ponciroli, "Description of Sensor Assignment Optimization Method as Deployed on a Multi-Node Cluster" in report ANL/NSE-20-13, Argonne National Laboratory, Lemont, IL, March 31, 2020.

[4] T. N. Nguyen, T. Downar and R. Vilim, "A probabilistic model-based diagnostic framework for nuclear engineering systems," Annals of Nuclear Energy, vol. 149, p. 107767, 2020.

[5] T. N. Nguyen, "Model-Based Diagnostic Frameworks for Fault Detection and System Monitoring in Nuclear Engineering Systems (Doctoral dissertation)," University of Michigan, Ann Arbor, 2020.

[6] J. Nocedal and S. Wright, Numerical Optimization, New York: Springer, 2000.

[7] M. Kochenderfer and T. Wheeler, Algorithms for Optimization, Cambridge, MA: The MIT Press, 2019.

[8] A. Mahajan, S. Leyffer, J. Linderoth and J. Luedtke, "Minotaur: a mixed-integer nonlinear optimization toolkit," Mathematical Programming Computation, vol. 13, p. 301-338, 2021.

[9] S. Sivanandam and S. Deepa, Introduction to Genetic Algorithms, New York, NY: Springer, 2008.

[10] F.-M. D. Rainville, F.-A. Fortin and M.-A. Gardner, "DEAP: A Python Framework for Evolutionary Algorithms," in The 14th Annual Conference Companion on Genetic and Evolutionary Computation, Philadelphia, Pennsylvania, USA, 2012.

[11] "multiprocessing," [Online]. Available: https://docs.python.org/3/library/multiprocessing.html.

[12] "Pytest," [Online]. Available: https://docs.pytest.org/en/6.2.x/index.html.

[13] Y. Liu, T. N. N. Brendan Kochunas, H. Ley and R. Vilim, "A Parallel Capability Using Genetic Algorithm for Sensor Assignment Optimization with Process-Constrained Data-Analytic Diagnosis," in 12th Nuclear Plant Instrumentation, Control and Human-Machine Interface Technologies (NPIC\&HMIT 2021), Virtual Meeting , 2021.

[14] Omega Engineering Inc., "Instrument Costs," [Online]. Available: https://www.omega.com/en-us/. 


\section{Argonne}

Nuclear Science and Engineering (NSE) Division

Argonne National Laboratory

9700 South Cass Avenue, Bldg. 208

Argonne, IL 60439

www.anl.gov 